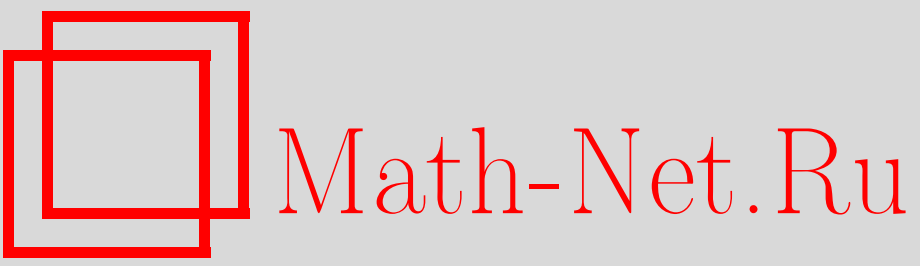

А. Г. Хованский, Многоугольники Ньютона, кривые на торических поверхностях и обращение теоремы Вейля, УМН, 1997, том 52, выпуск 6, 113-142

DOI: https://doi.org/10.4213/rm893

Использование Общероссийского математического портала Math-Net.Ru подразумевает, что вы прочитали и согласны с пользовательским соглашением

http://www.mathnet.ru/rus/agreement

Параметры загрузки:

IP : 18.209 .158 .208

26 апреля 2023 г., 15:45:35 


\title{
МНОГОУГОЛЬНИКИ НЬЮТОНА, КРИВЫЕ НА ТОРИЧЕСКИХ ПОВЕРХНОСТЯХ И ОБРАШЕНИЕ ТЕОРЕМЫ ВЕЙЛЯ
}

\author{
А. Г. ХовАнский \\ СОДЕРЖАНИЕ
}

Введение

$\S 1$. Многоугольники Ньютона, соотношение Паскаля и соотношение

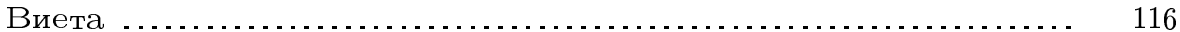

$\S 2$. Многоугольники Ньютона и числа Вейля … ......................... 119

$\S 3$. Параметризация одномерных орбит на торической поверхности $\ldots . . . \quad 122$

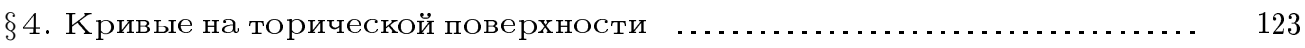

$\S 5$. Многоугольники без внутренних целых точек $\ldots \ldots \ldots \ldots \ldots \ldots \ldots \ldots \ldots . . \ldots \ldots$

$\S 6$. Многоугольник $\Delta_{D}$ с внутренней целой точкой $\ldots \ldots \ldots \ldots \ldots \ldots \ldots \ldots \ldots . \ldots \ldots$

$\S 7$. Многоугольник $\Delta_{D}$ без внутренних целых точек $\ldots \ldots \ldots \ldots \ldots \ldots \ldots \ldots . . \ldots 127$

$\S 8$. Мероморффные векторные функции на компактных кривых . . . . . . . . 130

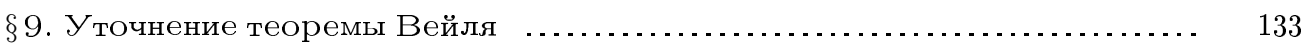

$\S 10$. Теорема Абеля и соотношение Виета ............................ 135

$\S 11$. Особенности характеристической кривой и многоугольники Нью-

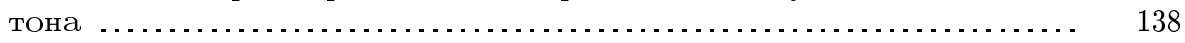

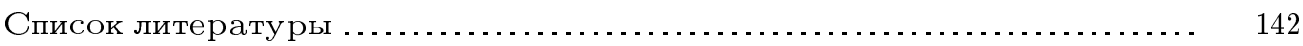

\section{Введение}

Для двух полиномов от одной переменной со старшими коэффициентами, равными единице, справедливо следующее тождество: произведение значений первого полинома по корням второго полинома с точностью до знака равно произведению значений второго полинома по корням первого.

Андрэ Вейль нашел далекое обобщение этого тождества. Оно применимо для любой пары ненулевых мероморфных функций на компактной комплексной кривой.

Приведем определения, нужные для формулировки теоремы Вейля. Пусть

$$
f=c_{1} u^{b_{1}}+\cdots, \quad g=c_{2} u^{b_{2}}+\cdots
$$

Работа выполнена во время визита в École Normale Supérieure при частичной поддержке Российского фонда фундаменталшных исследований (грант № 95-011-8701) и Канадского Гранта № 0GP0156833. Я признателен французским коллегам за гостеприимство. 
где $c_{1} \neq 0, c_{2} \neq 0$ - старшие члены рядов Лорана мероморфных функций $f$ и $g$ в окрестности точки $a$, и $u$ - локальный параметр такой, что $u(a)=0$. Tuпом ростка вектор-функции $(f, g)$ в точке $a$ назьвается несократимый целочисленньй вектор $\vec{n}=\left(n_{1}, n_{2}\right)$, пропорциональньй его вектору степени $\vec{b}=\left(b_{1}, b_{2}\right)$ с натуральным коэффициентом $k, \vec{b}=k \vec{n}$, которьй назьвается кратностью ростка вектор-функции. Приведенным числом Вейля ростка (*) назьвается число $c_{2}^{n_{1}} c_{1}^{-n_{2}}$, где $\left(n_{1}, n_{2}\right)-$ компоненты типа $\vec{n}$ этого ростка.

По компактной комплексной кривой Г и мероморфной вектор-функции $(f, g)$ на ней определим функцию $\mathrm{Mul}_{\Gamma f g}$ на произведении $\mathbb{Z}_{\mathrm{ir}}^{2} \times \mathbb{C}^{*}$ множества $\mathbb{Z}_{\mathrm{ir}}^{2}$ несократимых целочисленных векторов на плоскости на множество $\mathbb{C}^{*}$ ненулевых комплексных чисел. (Название функции происходит от слова "multiplicity" - кратность.) Функция $\mathrm{Mul}=\mathrm{Mul}_{\Gamma f g}$ принимает целые неотрицательные значения и равна нулю всюду кроме конечного числа точек. Ее значение на паре $(\vec{n}, c)$ по определению равняется суммарной кратности точек на комплексной кривой $\Gamma$, в которых росток $(f, g)$ имеет тип $\vec{n}$ и приведенное число Вейля $c$.

В этих терминах теорема Вейля формулируется следующим образом.

ТЕОРЕмА ВЕЙЛЯ.

$$
\prod(-c)^{\operatorname{Mul}(\vec{n}, c)}=1
$$

Степени дивизоров $f$ и $g$ на компактной кривой $Г$ равны нулю. В терминах функции $\mathrm{Mul}=\mathrm{Mul}_{\Gamma f g}$ эти соотношения принимают следующий и вид:

$$
\sum \operatorname{Mul}(\vec{n}, c) \vec{n}=0
$$

Результаты настоящей статьи основаны на следующем простом наблюдении: числа Вейля ростка $(f, g)$ и ростка $(F, G)$, где $F=f^{a_{11}} g^{a_{12}}, G=f^{a_{21}} g^{a_{22}}$ и $A=\left\{a_{i j}\right\}$ - целочисленная матрица с определителем 1 , равны. Это наблюдение подсказьвает, что теорема Вейля должна относиться к двумерной торической геометрии (матрища $A$ задает автоморфизм двумерного тора $\mathbb{C}^{* 2}$ ) и к теории многоугольников Ньютона. Мы показываем, что это действительно так. Прежде всего использование чисел Вейля упрощает и уточняет классическую теорему о многоугольниках Ньютона (см. §2). С другой стороны, использование многоугольников Ньютона позволяет дать очень простое доказательство теоремы Вейля - оно сводит теорему Вейля к формуле Виета для произведения корней полинома (см. $\S \S 1-2$ и $\S 9)$.

Обратима ли теорема Вейля? То есть верно ли, что для всякой функции Mul, удовлетворяющей условиям (1)-(2), найдется тройка $\Gamma, f, g$ такая, что $\mathrm{Mul}=\mathrm{Mul}_{\Gamma f g}$ ? В статье доказывается, что если у функции Mul больше двух характеристических векторов (т.е. таких векторов $\vec{n} \in \mathbb{Z}_{\mathrm{ir}}^{2}$, что функция $\operatorname{Mul}(\vec{n}, \cdot)$ на $\mathbb{C}^{*}$ не равна тождественно нулю), то ответ на этот вопрос положителен. Дается полное описание троек $\Gamma, f, g$ таких, что Mul $=\operatorname{Mul}_{\Gamma f g}$.

В исключительном случае, когда функщия $\mathrm{Mul}_{\Gamma f g}$ имеет два характеристических вектора, теорема Вейля допускает уточнение. Функция $\mathrm{Mul}=\mathrm{Mul}_{\Gamma f g}$ в этом случае обладает свойством возвратности

$$
\operatorname{Mul}(\vec{n}, c)=\operatorname{Mul}\left(-\vec{n}, c^{-1}\right) .
$$


В $\S 9$ дается простое независимое доказательство этого свойства. Уточненная теорема Вейля оказывается обратимой и в исключительном случае.

С тройкой $\Gamma, f, g$ связьвается некоторый многоугольник, назьваемьй многоугольником Ньютона. Этот многоугольник восстанавливается по функции $\mathrm{Mul}=\mathrm{Mul}_{\Gamma f g}$. Мы показьваем, что он играет ту же роль, что и обычньй многоугольник Ньютона (см. $\S 11)$.

Перейдем к другой теме статьи. Пусть $D$ - дивизор, лежаший на объединении $M_{\infty}$ одномерных орбит торической поверхности $M$. Существует ли кривая $\Gamma \subset M$, не проходящая через нульмерные орбиты поверхности $M$, для которой дивизор $D$ является дивизором пересечения кривой $\Gamma$ с объединением одномерных орбит $M_{\infty}$ ? Пространство таких кривых $Г$ мы будем обозначать через $\mathscr{R}(D)$, а дивизор $D$, для которого пространство $\mathscr{R}(D)$ не пусто, будем назьвать допустимым. В статье дивизоры $D$ кодируются при помощи некоторой функции $\mathrm{Mul}=\operatorname{Mul}_{D}$ на $\mathbb{Z}_{\mathrm{ir}}^{2} \times \mathbb{C}^{*}$. Условие допустимости дивизора $D$, как оказьвается, в точности совпадает с условием существования тройки $\Gamma, f, g$ такой, что $\mathrm{Mul}=\mathrm{Mul}_{\Gamma f g}$.

В $\S \S 6-7$ описывается общая кривая из пространства $\mathscr{R}(D)$. С допустимым дивизором $D$ связывается некоторый многоугольник $\Delta_{D}$. Задача описания общей кривой из пространства $\mathscr{R}(D)$ эквивалентна исследованию общего уравнения $P=0$ с данным многоугольником Ньютона $\Delta(P)=\Delta_{D}$ и с фиксированными коэффициентами при мономах, соответствующих точкам на гранище многоугольника $\Delta$.

Исследование общего уравнения с данным многогранником Ньютона - традиционная задача. Начальные данные в этой задаче (многогранник Ньютона) дискретны. В нашей задаче кроме дискретных данных (многоугольник Ньютона) фигурируют непрерьвные данные (коэффициенты на границе). Решение нашей задачи, в частности, включает в себя описание всех кривых $P=0$, многоугольник Ньютона которых не содержит внутренних целых точек. Его удается провести потому, что многоугольников без внутренних целых точек не так уж и много (см. §5).

Среди условий допустимости дивизора $D$ есть дискретное условие и непрерывное условие (условие Виета, см. §4). В §10 приводится вариант теоремы Абеля, даюший независимое объяснение условия Виета.

Пусть $P$ - полином Лорана на комплексной плоскости. В статье по полиному Лорана $P$ определяется некоторая функция $\mathrm{Mul}=\mathrm{Mul}_{P}$ на пространстве $\mathbb{Z}_{\mathrm{ir}}^{2} \times \mathbb{C}^{*}$. Сушествует ли для заданной функции $\mathrm{Mul}$ полином Лорана $P$ такой, что $\mathrm{Mul}=\mathrm{Mul}_{P}$ ?

Именно эта несложная задача играет ключевую роль в настоящей статье. Оказывается, что условия на функцию Mul для существования полинома Лорана $P$ такого, что $\mathrm{Mul}=\mathrm{Mul}_{P},-$ это ровно те же условия, о которых говорилось вьше (т.е. условия сушествования тройки $\Gamma, f, g$ такой, что Mul $=\operatorname{Mul}_{\Gamma f g}$, или условие допустимости дивизора $D$ такого, что $\mathrm{Mul}=\mathrm{Mul}_{D}$ ).

Задача сушествования полинома Лорана $P$ такого, что $\mathrm{Mul}=\mathrm{Mul}_{P}$, допускает полное решение, используюшее лиш элементарную геометрию плоскости (условие Паскаля для выпуклых многоугольников) и элементарную алгебру (формулу Виета для произведения корней полинома). С решения этой задачи мы и начинаем статью.

Одно терминологическое замечание: под словами "многогранник" и "многоугольник" подразумеваются вьпукльй многогранник и выпукльй многоугольник. 


\section{$\S$ 1. Многоугольники Ньютона, соотношение Паскаля и соотношение Виета}

Пусть $P(x, y)$ - полином Лорана на комплексной плоскости и $\Delta=\Delta(P)$ - его многоугольник Ньютона, лежаший в плоскости показателей. Плоскость показателей $\mathbb{R}^{2}$ будем считать ориентированной. (Ее ориентация задается порядком переменных $x, y$.) $\mathrm{B}$ ней выделена решетка целочисленных показателей. Двойственная плоскость $\mathbb{R}^{2 *}$, следовательно, тоже ориентирована, и в ней выделена двойственная решетка, которую мы будем назьвать решеткой степеней. Обозначим через $\mathbb{Z}_{\mathrm{ir}}^{2}$ подмножество в решетке степеней, состоящее из несократимых ненулевых векторов.

С каждым вектором $\vec{n} \in \mathbb{Z}_{\mathrm{ir}}^{2}$ связана ориентация ортогональной прямой к вектоpy $\vec{n}$, лежашей в плоскости показателей. Именно, прямая ориентируется как граница области, в которой скалярное произведение с вектором $\vec{n}$ положительно. Так, при обычной ориентации плоскости с внутренней нормалью $\vec{n}$ к стороне двумерного многоугольника $\Delta$ связана ее ориентация, соответствующая движению по гранище многоугольника против часовой стрелки.

Для каждого вектора $\vec{n} \in \mathbb{Z}_{\mathrm{ir}}^{2}$ обозначим через $\Delta^{\vec{n}}$ сторону или вершину многоугольника $\Delta$, на которой достигает минимума скалярное произведение с вектором $\vec{n}$.

Для каждого целочисленного многоугольника $\Delta$ определена функция Lengh ${ }_{\Delta}$ на $\mathbb{Z}_{\text {ir }}^{2}$, сопоставляющая вектору $\vec{n}$ целочисленную длину грани $\Delta^{\vec{n}}$. По определению челочисленная длина вершины равна нулю, а целочисленная длина стороны, содержащей $(m+1)$ целую точку, равна $m$.

Согласно знаменитой теореме Минковского, многомерный многогранник однозначно с точностью до параллельного переноса восстанавливается по функции $S(\vec{n})$, сопоставляющей нормалям $\vec{n}$ площади $S$ соответствующих им граней. Многогранник существует, если и только если вьполнено условие Паскаля $\sum S(\vec{n}) \vec{n}=0$.

Следуюшая простая лемма является двумерным целочисленным вариантом теоремы Минковского. Пусть Lengh - функция на $\mathbb{Z}_{\mathrm{ir}}^{2}$ со значениями в целых неотрицательных числах.

ЛЕмма (целочисленный вариант двумерной теоремы Минковского). Для ненулевой функиии Lengh существует челочисленный многоугольник $\Delta$ такой, что Lengh $=$ Lengh $_{\Delta}$, если и только если функция отлична от нуля лишь на конечном множестве и выполнено условие Паскаля

$$
\sum \operatorname{Lengh}_{\Delta}(\vec{n}) \vec{n}=0 .
$$

Многоугольник $\Delta$ определяется по функиии Lengh однозначно с точностью до параллельного переноса.

ДокАЗАТЕльСтво. Покажем, как построить многоугольник по функции Lengh, удовлетворяюший условию Паскаля. Зададим структуру комплексной прямой на плоскости $\mathbb{R}^{2}$ и отождествим тем самым $\mathbb{R}^{2}$ и $\mathbb{R}^{2 *}$. Рассмотрим конечное множество векторов из $\mathbb{Z}_{\mathrm{ir}}^{2}$, для которых функция Lengh не равна нулю. Занумеруем вектора этого множества в порядке, в котором они встречаются при вращении линейки против часовой стрелки вокруг начала координат. Положим $l_{\vec{n}_{j}}=(-i) \operatorname{Lengh}\left(\vec{n}_{j}\right)\left(\vec{n}_{j}\right)$, где $i$ - мнимая единица. Построим из векторов $l_{\vec{n}}$ ломаную, последовательно прикладывая начало следуюшего вектора к концу предыдущего. Условие замыкания ломаной 
эквивалентно выполнению условия Паскаля. Построенная ломаная является границей искомого многоугольника. Остальные утверждения леммы доказываются столь же просто.

Для каждого вектора $\vec{n} \in \mathbb{Z}_{\mathrm{ir}}^{2}$ по полиному Лорана $P$ определим следуюший полином $P_{\vec{n}}$ от одной переменной. Если $\Delta^{\vec{n}}$ является вершиной $Q$, то полином $P_{\vec{n}}$ определяется как константа, равная коэффициенту в полиноме Лорана $P$ при мономе, соответствуюшем вершине $Q$. Пусть теперь $\Delta^{\vec{n}}$ является стороной $l$ многоугольника Ньютона $\Delta$.

Вершины $A, B$ на этой стороне $l$ будем назьвать, соответственно, старией и младшей, если движение по стороне $l$ от точки $A$ к точке $B$ соответствует ориентации стороны $l$, связанной с вектором $\vec{n}$. Пусть сторона $l$ имеет целочисленную длину $m$, т.е. содержит $(m+1)$ целую точку. Занумеруем целые точки на стороне $l$ числами от 0 до $m$, начиная с младшей вершины $B$. Пусть коэффициент в полиноме Лорана $P$ при мономе, соответствуюшем $i$-й целой точке, равен $c_{i}$. Определим полином $P_{\vec{n}}$ формулой $P_{\vec{n}}(\xi)=\sum_{i=0}^{m} c_{i} \xi^{i}$. Таким образом, полином $P_{\vec{n}}$ имеет степень, равную целочисленной длине $m$ стороны $l$. Его старший коэффициент равен коэффициенту в полиноме Лорана $P$ при вершине $A$. Свободньй член полинома $P_{\vec{n}}$ равен коэффищиенту полинома Лорана $P$ при вершине $B$.

Каждому полиному Лорана $P(x, y)$ соответствует функция $\mathrm{Mul}_{P}$ на $\mathbb{Z}_{\mathrm{ir}}^{2} \times \mathbb{C}^{*}$, сопоставляюшая вектору $\vec{n}$ и комплексному числу $c$ кратность, с которой число $c$ является корнем полинома $P_{\vec{n}}(\xi)$. По функции $\mathrm{Mul}_{P}$ с точностью до множителя восстанавливается каждый из полиномов $P_{\vec{n}}$. В частности, восстанавливаются степени этих полиномов, т.е. функция Lengh ${ }_{\Delta(P)}$. Поэтому следуюший вопрос является алгебраическим вариантом вопроса Минковского: существует ли для заданной функции Mul: $\mathbb{Z}_{\mathrm{ir}}^{2} \times \mathbb{C}^{*} \rightarrow \mathbb{N}_{+}$полином Лорана $P$, для которого Mul $=\operatorname{Mul}_{P} ?$ Ниже мы приведем полный ответ на этот вопрос.

Скажем, что вектор $\vec{n}_{j} \in \mathbb{Z}_{\mathrm{ir}}^{2}$ является характеристическим для функции Mul, если ограничение $\operatorname{Mul}(\vec{n}, \cdot)$ этой функции на $\mathbb{C}^{*}$ не равно тождественно нулю.

Теорема (алгебраический аналог двумерной теоремы Минковского). Для ненулевой функиии Mul существует полином Лорана $P$ такой, что $\mathrm{Mul}=\mathrm{Mul}_{P}$, если и только если функиия $\mathrm{Mul}$ имеет конечное число характеристических векторов и

1) (одномерньй случай) если характеристических векторов не более двух, то выполняется условие возвратности

$$
\operatorname{Mul}(\vec{n}, c)=\operatorname{Mul}\left(-\vec{n}, c^{-1}\right) ;
$$

2) (двумерный случай) если характеристических векторов более двух, то выполняются

а) условие Паскаля: $\sum \operatorname{Mul}(\vec{n}, c) \vec{n}=0$,

b) условие Buета: $\prod(-c)^{\operatorname{Mul}(\vec{n}, c)}=1$.

ДокАЗАТЕЛЬСТво. Начнем с одномерного случая.

Прежде всего, из условия возвратности, очевидно, вытекает как условие Паскаля, так и условие Виета. Кроме того, если ненулевая функция Mul обладает свойством 
возвратности, то она имеет ровно два характеристических вектора $\vec{n}_{1}$ и $\vec{n}_{2}$, связанных соотношением $\vec{n}_{1}+\vec{n}_{2}=0$.

Если многоугольник Ньютона полинома Лорана $P$ является отрезком $l$, то полином $P_{\vec{n}}$ не равен константе ровно для двух векторов, а именно, для векторов $\vec{n}_{1}, \vec{n}_{2}$, ортогональных отрезку $l$. В этом случае имеем очевидные соотношения $\vec{n}_{1}=-\vec{n}_{2}$ и $\xi^{m} P_{\vec{n}_{1}}\left(\xi^{-1}\right)=P_{\vec{n}_{2}}(\xi)$, где $m$ - целочисленная длина отрезка $l$. Отсюда вытекает условие возвратности $\operatorname{Mul}_{P}\left(\vec{n}_{1}, c\right)=\operatorname{Mul}_{P}\left(-\vec{n}_{1}, c^{-1}\right)$. Обратно, пусть функция Mul возвратна и имеет два характеристических вектора $\vec{n}_{1}$ и $\vec{n}_{2}, \vec{n}_{1}+\vec{n}_{2}=0$. Многоугольник, построенный по функции $\operatorname{Lengh}(\vec{n})=\sum \operatorname{Mul}(\vec{n}, c) \vec{n}$ будет отрезком, ортогональным вектору $\vec{n}_{1}$, целочисленная длина которого равна Lengh $\left(\vec{n}_{1}\right)=\sum \operatorname{Mul}\left(\vec{n}_{1}, c\right)$.

Рассмотрим полином Лорана $P$, многоугольник Ньютона которого совпадает с построенным отрезком, а полином $P_{\vec{n}_{1}}(\xi)$ равен $c_{0} \prod(\xi-c)^{\operatorname{Mul}\left(\vec{n}_{1}, c\right)}$. Эти условия определяют полином Лорана $P$.

Функция $\operatorname{Mul}(P)$ совпадает с функцией Mul, так как полином $P_{\vec{n}_{2}}(\xi)=\xi^{\operatorname{Lengh}\left(\vec{n}_{1}\right)} \times$ $P_{\vec{n}_{1}}\left(\xi^{-1}\right)$ имеет корни, обратные к корням полинома $P_{\vec{n}_{1}}$. В одномерном случае теорема доказана в обе стороны.

Докажем теперь теорему для двумерного случая. Пусть полином Лорана $P$ имеет двумерньй многоугольник Ньютона. Для стороны $l_{\vec{n}}$ многоугольника Ньютона $\Delta \mathrm{c}$ внутренней нормалью $\vec{n}$ выполнено следуюшее соотношение:

$$
\prod_{c}(-c)^{\mathrm{Mul}_{P}(\vec{n}, c)}=\frac{P_{B}}{P_{A}}
$$

где $A$ и $B$ - соответственно, старшая и младшая вершины на стороне $l$, а $P_{A}, P_{B}$ - коэффициенты в полиноме Лорана $P$, соответствуюшие этим вершинам. Действительно, написанное соотношение является формулой Виета для произведения корней полинома $P_{\vec{n}}$. Произведение $\prod(-c)^{\operatorname{Mul}_{P}(\vec{n}, c)}$ равно единице, так как каждая вершина многоугольника Ньютона является младшей вершиной для одной стороны и старшей - для другой.

Докажем теперь обратное утверждение. В случае 2) условие Паскаля постулируется. Поэтому по функции Mul можно построить многоугольник. Ясно, что он будет двумерным. Знание функции $\operatorname{Mul}(\vec{n}, c)$ при фиксированном векторе $\vec{n}$ позволяет восстановить все коэффициенты полинома Лорана $P$ на стороне $l_{\vec{n}}$ с точностью до произвольного множителя $c_{0}, P_{\vec{n}}(\xi)=c_{0} \prod(\xi-c)^{\operatorname{Mul}(\vec{n}, c)}$.

Покажем, что вьполнение условия Виета гарантирует возможность согласованного выбора произвольных множителей в полиномах $P_{\vec{n}}$. Действительно, стороны в двумерном многоугольнике пересекаются лишш по вершинам. Начнем последовательно обходить стороны $l_{1}, \ldots, l_{N}$ по границе многоугольника $\Delta$ и выписьвать полиномы $P_{\vec{n}_{1}}, \ldots, P_{\vec{n}_{N}}$, соответствуюшие внутренним нормалям к этим сторонам. Обозначим через $V_{i}$ произведение $\prod(-c)^{\operatorname{Mul}\left(\vec{n}_{i}, c\right)}$. Фиксируем множитель $c_{0}=Q$ в старшей вершине стороны $l_{1}$ и положим $P_{\vec{n}_{1}}(\xi)=Q \prod(\xi-c)^{\operatorname{Mul}\left(\vec{n}_{1}, c\right)}$. Тогда коэффициент в младшей вершине этой стороны будет равен $Q V_{1}$. Младшая вершина стороны $l_{1}$ является старшей для стороны $l_{2}$. Поэтому полином $P_{\vec{n}_{2}}(\xi)$ должен быть равен $Q V_{1} \prod(\xi-c)^{\operatorname{Mul}\left(\vec{n}_{2}, c\right)}$. Коэффициент при младшей вершине стороны $l_{2}$ будет равен $Q V_{1} V_{2}$. Двигаясь по границе многоугольника, мы дойдем до вершины, с которой 
начинали. При этом мы не получим противоречия, так как $Q V_{1} \cdots V_{N}=Q$, ибо произведение $\prod(-c)^{\operatorname{Mul}(\vec{n}, c)}$ по условию Виета равно единице. Итак, мы восстановили с точностью до общего множителя коэффициенты полинома Лорана $P$ на границе многоугольника $\Delta$. Выбирая произвольным образом коэффищиенты при мономах, соответствующих внутренним точкам многоугольника $\Delta$, получим полином $P$ такой, что $\mathrm{Mul}=\operatorname{Mul}(P)$. Теорема доказана.

Формулируемое ниже следствие имеет довольно неожиданную переформулировку в геометрии торических поверхностей (см. теорему 2 из $§ 10)$.

СЛЕДСТВИЕ. Для всякого целочисленного многоугольника $\Delta$ найдется полином Лорана $Q$, многоугольник Ньютона которого равен $\Delta$, обладающий следующим свойством: все полиномы $Q_{\vec{n}}, \vec{n} \in \mathbb{Z}_{\mathrm{ir}}^{2}$, не имеют корней, отличных om $(-1)$.

Следствие непосредственно вытекает из теоремы. Впрочем, оно имеет даже более простое доказательство.

ДоКАЗАТЕЛЬСТВо С ЛЕДСТВИЯ. Если минимум скалярного произведения с вектором $\vec{n} \in \mathbb{Z}_{\mathrm{ir}}^{2}$ достигается на некоторой стороне $l$ многоугольника $\Delta$ и целочисленная длина стороны $l$ равна $m(l)$, то положим $Q_{\vec{n}}(\xi)=(1+\xi)^{m(l)}$. Если же минимум достигается в вершине, то положим $Q_{\vec{n}}(\xi) \equiv 1$. Как старший, так и младший коэффициенты полинома $(1+\xi)^{m}$ равны 1 . Поэтому если многоугольник $\Delta$ двумерен, то, написав на каждой его стороне $l$ полином $(1+\xi)^{m(l)}$, мы не получим противоречия: стороны многоугольника пересекаются лишш по вершинам, и в каждой вершине будет написан коэффициент, равньй 1. Если же многоугольник состоит из одного отрезка, то мы не получим противоречия, так как полином $(1+\xi)^{m}$ возвратен, $\xi^{m}\left(1+\xi^{-1}\right)^{m}=(\xi+1)^{m}$. Во внутренних целых точках многоугольника $\Delta$ можно написать любые коэффициенты. Мы получим искомьй полином Лорана $Q$.

Подведем итог. Функцию Mul будем назьвать допустимой, если она удовлетворяет условию теоремы. По допустимой функции Mul однозначно (с точностью до параллельного переноса) строится многоугольник $\Delta=\Delta(\mathrm{Mul})$. По допустимой функции Mul у полинома Лорана $P$ такого, что $\mathrm{Mul}_{P}=\mathrm{Mul}$, однозначно (с точностью до одновременного умножения на ненулевую константу) восстанавливаются все коэффициенты при мономах, соответствуюших точкам на гранище многоугольника $\Delta$. Коэффициенты при внутренних мономах выбираются произвольным образом. Поэтому полиномы Лорана $P$, для которых $\mathrm{Mul}_{P}=\mathrm{Mul}$, являются множеством ненулевых векторов в комплексном линейном пространстве, размерность которого равна $B(\Delta)+1$, где $B(\Delta)$ - число внутренних целых точек многоугольника $\Delta=\Delta(\mathrm{Mul})$.

\section{§ 2. Многоугольники Ньютона и числа Вейля}

Пусть $\Gamma$ - росток аналитической кривой в точке $a$ и $u$-локальньй параметр, $u: \Gamma \rightarrow$ $C$, такой, что $u(a)=0$. Рассмотрим росток $(f, g)$ мероморфной функции на Г. Пусть

$$
f=c_{1} u^{b_{1}}+\cdots, \quad g=c_{2} u^{b_{2}}+\cdots,
$$


где $c_{1} \neq 0, c_{2} \neq 0$ - старшие члены рядов Лорана этих функций. (Мы предполагаем сейчас и на протяжении всей статьи, что ни росток $f$, ни росток $g$ не обрашаются тождественно в нуль. Мы также всегда будем предполагать, что росток $(f, g)$ не является ростком постоянной вектор-функции.)

Числом Вейля ростка вектор-функции $(f, g)$ назовем число

$$
\{f, g\}_{a}=(-1)^{b_{1}+b_{2}+b_{1} b_{2}} c_{2}^{b_{1}} c_{1}^{-b_{2}} .
$$

Несложно проверить, что число $\{f, g\}_{a}$ определено корректно, т.е. не зависит от выбора локального параметра $u$.

Числа Вейля встречаются в теореме Вейля (см. 9 ), тесно связанной с материалом настояшей статьи.

Приведем определения еше нескольких инвариантов ростка (1) вектор-функции $(f, g)$. Типом ростка вектор-функции $(f, g)$ назьвается несократимый целочисленный вектор $\vec{n}=\left(n_{1}, n_{2}\right)$, пропорциональньй его вектору степени $\vec{b}=\left(b_{1}, b_{2}\right)$ с натуральньм коэффициентом $k, \vec{b}=k \vec{n}$, которьй назьвается кратностью ростка вектор-функции $(f, g)$. Приведеннылм числом Вейля $[f, g]_{a}$ ростка вектор-функции $(f, g)$ назьвается число $[f, g]=c_{2}^{n_{2}} c_{1}^{-n_{1}}$, где $\left(n_{1}, n_{2}\right)$ - компоненты типа $\vec{n}$ этого ростка.

Непосредственно из определений выводится следующая

ЛЕмма 1. Число Вейля следующим образом выражается через приведенное число Вейля и кратность ростка:

$$
\{f, g\}_{a}=\left(-[f, g]_{a}\right)^{k} .
$$

Инварианты ростка мероморфной функции, с которьми мы имеем дело, сохраняются при степенных преобразованиях. Пусть $A=\left\{a_{i j}\right\}$ - унимодулярная матрица (т.е. матрица с целыми коэффициентами и определителем, равным единице) размера $2 \times 2$. С матрицей $A$ связано степенное преобразование вектор-функции $(f, g)$. Росток $(f, g)$ при этом преобразовании переходит в росток $(F, G)$, где

$$
F=f^{a_{11}} g^{a_{12}} \quad \text { и } \quad G=f^{a_{21}} g^{a_{22}} .
$$

ЛЕмма 2. Для всякой унимодулярной матриць А тип ростка вектор-функции $(F, G)$ равен $A \vec{n}$, где $\vec{n}$ - тип ростка вектор-функиии $(f, g)$. Кратность, приведенное число Вейля и число Вейля для ростка вектор-функиии $(F, G)$ такие же, как для ростка вектор-функции $(f, g)$.

Лемма 2 проверяется непосредственным вычислением. Она подсказьвает, что числа Вейля должны быть связаны с теорией многоугольников Ньютона и с двумерной торической геометрией.

В статье мы покажем, что это действительно так. Прежде всего, использование чисел Вейля упрощает формулировку приведенной ниже классической теоремы о многоугольниках Ньютона.

Пусть алгебраическая кривая $Г$ лежит в торе $\mathbb{C}^{* 2}$ с координатньми функщиями $x$ и $y$. На нормализации $\bar{\Gamma}$ этой кривой определены две мероморфные функции $x$ и $y$, мероморфно отображаюшие кривую $\bar{\Gamma}$ в тор $\mathbb{C}^{* 2}$ на кривую $\Gamma \subset \mathbb{C}^{* 2}$. Следуюшая теорема восходит к Ньютону. 
Tеорема 1 (о многоугольниках Ньютона). Пусть кривая $Г$ определена в $\mathbb{C}^{* 2}$ уравнением $P(x, y)=0$ и не имеет кратных компонент. На нормализации $\bar{\Gamma}$ этой кривой существуют точки, в которьих вектор-функция $(x, y)$ имеет тип $\vec{n} \neq 0$, если и только если на многоугольнике Ньютона $\Delta(P)$ минимум скалярного произведения с вектором $\vec{n}$ достигается на стороне этого многоугольника. Более того, посчитанное с учетом кратностей число точек, в которых вектор-функиия $(x, y)$ имеет тип $\vec{n}$, а ее приведенное число Вейля является заданныцм числом $\xi_{0}$, равно порядку $\operatorname{ord}_{\xi_{0}} P_{\vec{n}}$ полинома $P_{\vec{n}}$ в точке $\xi_{0}$.

ДокАЗАТЕЛЬСТво. Сделаем степенное преобразование плоскости $x, y$ при помоши унимодулярной матрицы $A$, переводящей несократимьй вектор $\vec{n}$ в вектор $(1,0)$. При таком преобразовании ни приведенные числа Вейля, ни кратности ростков вектор-функции $(x, y)$ на кривой $\bar{\Gamma}$ не меняются (см. лемму 1$)$. Нас интересуют такие точки на кривой $\bar{\Gamma}$, в окрестности которых функции $x$ и $y$ имеют вид

$$
\begin{aligned}
& x=c_{1} u^{k}+\cdots, \\
& y=c_{2}+\cdots,
\end{aligned}
$$

где $c_{1} c_{2} \neq 0, k>0$.

Допустим, что минимум скалярного произведения с вектором $(1,0)$ достигается на стороне $l$ многоугольника $\Delta(P)$. Параллельно перенесем многоугольник $\Delta(P)$ так, чтобы младшая вершина на стороне $l$ находилась в начале координат. Параллельный перенос соответствует умножению полинома Лорана $P$ на моном и не меняет кривой $\Gamma$, определенной в торе $\mathbb{C}^{* 2}$ уравнением $P=0$.

После такого преобразования ограничение полинома Лорана $P$ на ось $y$ при $y \neq 0$ определено. При этом полином $P(0, y)$ совпадает с определенным вьше полиномом $P_{\vec{n}}(\xi)$ при $\xi=y$. С одной стороны, в точке $\left(0, \xi_{0}\right)$ кратность пересечения оси $y$ с замыканием кривой $\Gamma \subset \mathbb{C}^{* 2}$ равна кратности корня $\xi_{0}$ полинома $P(0, y)=P_{\vec{n}}(y)$. С другой стороны, эта кратность равна числу точек на нормализованной кривой $\bar{\Gamma}$, около котоpux

$$
\begin{aligned}
& x=c_{1} u^{k}+\cdots, \\
& y=\xi_{0}+\cdots,
\end{aligned}
$$

посчитанных с учетом их кратностей $k$. Что и нужно было доказать.

Если минимум скалярного произведения с вектором $\vec{n}$ достигается в вершине многоугольника $\Delta(P)$, то теорема доказьвается так же (и даже проще: в этом случае полином $P_{\vec{n}}$ является константой и вообще не имеет корней).

Теорема о многоугольниках Ньютона непосредственно обобщается на случай алгебраических кривых, имеюших кратные компоненты. Полной кратностью для ростка (1) вектор-функщии на компоненте алгебраической кривой кратности $\mu$ назовем число $k \mu$, где $k$ - кратность для ростка (1).

Tеорема 1' (о многоугольниках Ньютона). На нормализации $\bar{\Gamma}$ кривой $\Gamma$, заданной в $\mathbb{C}^{* 2}$ уравнением $P(x, y)=0$, существуют точки, в которых вектор-функиия $(x, y)$ имеет тип $\vec{n} \neq 0$, если и только если на многоугольнике 
Ньютона $\Delta(P)$ минимум скалярного произведения с вектором $\vec{n}$ достигается на стороне этого многоугольника. Более того, посчитанное с учетом полньх кратностей число точек, в которых вектор-функиия $(x, y)$ имеет тип $\vec{n}$, а ее приведенное число Вейля является заданным числом $\xi_{0}$, равно порядку $\operatorname{ord}_{\xi_{0}} P_{\vec{n}}$ полинома $P_{\vec{n}}$ в точке $\xi_{0}$.

Теорема 1' автоматически вытекает из теоремы 1. Дело в том, что полином Лорана $P$ разлагается в произведение неприводимых полиномов Лорана, для которых справедлива теорема 1.

\section{§ 3. Параметризация одномерных орбит на торической поверхности}

Многогранники Ньютона, как известно, теснейшим образом связаны с торическими компактификациями тора [4].

Двумерная ситуация специальна. Група $\mathbb{C}^{*}$ имеет только один нетривиальньй автоморфизм (при котором точка $t$ переходит в точку $t^{-1}$ ). Специфика двумерного тора заключается в существовании одновременно естественного выбора параметризации на каждой его связной фактор-групе размерности 1. Эта параметризация зависит лишь от ориентации плоскости однопараметрических групп и меняется на противоположную при смене ориентации. Определим эту параметризацию. Фиксируем ориентацию плоскости однопараметрических. Пусть $O_{\vec{b}}$ - однопараметрическая подгруппа в $\mathbb{C}^{* 2}$, соответствуюшая вектору $\vec{b}=\left(b_{1}, b_{2}\right), \vec{b} \neq 0$. (Такая группа определяется гомоморфизмом стандартной групш $\mathbb{C}^{*}$ в групу $\mathbb{C}^{* 2}$, переводящим точку $\tau$ в точку $\tau^{\vec{b}}=\left(\tau^{b_{1}}, \tau^{b_{2}}\right)$.) Обозначим через $\pi_{\vec{b}}$ проекцию группы $\mathbb{C}^{* 2}$ на фактор-группу групшы $\mathbb{C}^{* 2}$ по подгруппе $O_{\vec{b}}$.

ОПРЕДЕЛЕНИЕ. Параметризацию $t$ фактор-групшы $\mathbb{C}^{* 2} / O_{\vec{b}}$ будем назьвать nараметризаиией, согласованной с ориентацией плоскости однопараметрических, если для любого целочисленного вектора $\vec{m}$ такого, что пара векторов $(\vec{b}, \vec{m})$ задает правильную ориентащию плоскости однопараметрических, справедливо равенство

$$
\lim _{\tau \rightarrow 0} t\left(\pi_{\vec{b}}\left(\tau^{\vec{m}}\right)\right)=0
$$

Несложно проверить, что параметризация, согласованная с ориентацией плоскости однопараметрических, действительно сушествует. Опишем ее в координатах. Рассмотрим стандартньй двумерньй тор $\mathbb{C}^{* 2}$ с координатньми функциями $(x, y)$ и со стандартной ориентацией плоскости однопараметрических. Пусть $\vec{b}=\left(b_{1}, b_{2}\right)-$ не равный нулю целочисленный вектор, $\vec{n}=\left(n_{1}, n_{2}\right)$ - несократимый целочисленный вектор такой, что $\vec{b}=k \vec{n}$, где $k$ - натуральное число, и $\pi_{\vec{b}}-$ проекция $\mathbb{C}^{* 2}$ на фактор-группу $\mathbb{C}^{* 2} / O_{\vec{b}}$.

Легко проверяется следуюшая

Лемма. Отображение, сопоставляющее точке $c \in \mathbb{C}^{* 2}$ параметр $t$ точки $\pi_{\vec{b}}(c)$ при описанной выше параметризачии фактор-группь, задается формулой $t=c_{2}^{n_{1}} c_{1}^{-n_{2}}$, әде $c=\left(c_{1}, c_{2}\right), \vec{n}=\left(n_{1}, n_{2}\right)$.

Фиксация изоморфизма одномерных фактор-груп тора $\mathbb{C}^{* 2}$ с группй $\mathbb{C}^{*}$ имеет следующее последствие. 
УТВЕРЖДЕНИЕ 1. На всякой торической поверхности существует естественная параметризачия каждой одномерный орбиты, зависящая лишь от ориентации плоскости однопараметрических.

ДокАЗАТЕльство. Действительно, пусть $M_{\infty}^{\vec{n}}$ - одномерная орбита, соответствующая лучу, порожденному вектором $\vec{n}$ в веере этой поверхности (см. [3], [7]). Точки тора $\mathbb{C}^{* 2}$ стремятся к этой орбите при действии однопараметрической $t^{\vec{n}}$ при $t \rightarrow 0$. Орбита $M_{\infty}^{\vec{n}}$ естественно изоморфна фактор-группе тора $\mathbb{C}^{* 2}$ по подгрупе $t^{\vec{n}}$. Описанная выше параметризация этой фактор-группы задает естественную параметризацию орбиты.

Связь этой торической конструкции с числами Вейля следующая. Пусть $f=c_{1} u^{b_{1}}+\cdots, g=c_{2} u^{b_{2}}+\cdots$ - росток мероморфного отображения кривой $(\Gamma, a)$ в тор $\mathbb{C}^{* 2}$. Пусть $\vec{b}=k \vec{n}, k>0$, и $M^{\vec{n}} \supset \mathbb{C}^{* 2}$ - торическая поверхность, веер которой является лучом, порожденным вектором $\vec{n}$. Поверхность $M^{\vec{n}}$ содержит ровно одну одномерную орбиту $M_{\infty}^{\vec{n}}$. Порядок компонент $f$ и $g$ в вектор-функции $(f, g)$ фиксирует ориентацию плоскости и, следовательно, фиксирует параметризацию одномерной орбиты $M_{\infty}^{\vec{n}}$.

УТВеРЖДЕНИЕ 2. Росток мероморфного отображения $(f, g): \Gamma \rightarrow \mathbb{C}^{* 2}$ продолжается до ростка аналитического отображсения $(\widetilde{f}, \widetilde{g}): \Gamma \rightarrow M^{\vec{n}}$. Точка а при этом аналитическом отображении переходит в точку одномерной орбить $M_{\infty}^{\vec{n}}$ с параметром $t$, равнымм приведенному числу Вейля $[f, g]_{a}$. Образ кривой Г пересекается с орбитой $M_{\infty}^{\vec{n}}$ с кратностью $k$, равной кратности ростка $(f, g)$.

ДокАЗАТЕЛЬСтво. Действительно, при $u \rightarrow 0$ точка $\left(c_{1} u^{b_{1}}+\cdots, c_{2} u^{b_{2}}+\cdots\right)$ будет стремиться к точке на орбите $M_{\infty}^{\vec{n}}$, параметр которой по лемме равен $c_{2}^{n_{1}} c_{1}^{-n_{2}}$. Это число равно приведенному числу Вейля. Остальные факты из утверждения легко проверяются.

\section{$\S 4$. Кривые на торической поверхности}

Пусть $M$ - компактная торическая поверхность, возможно, имеюшая особенности в точках, являющихся нульмерньми орбитами. Пусть на объединении $M_{\infty}$ одномерных орбит поверхности $M$ фиксировано конечное число точек с заданньми положительными кратностями. Сушествует ли кривая на поверхности $M$, не проходящая через нульмерные орбиты и пересекающая одномерные орбиты в фиксированных точках с заданными кратностями? Здесь предлагается полньй ответ на этот вопрос.

Для формулировки ответа, прежде всего, закодируем дивизоры $D$, носители которых лежат в объединении одномерных орбит поверхности $M$, при помоши определяемой ниже функции

$$
\operatorname{Mul}_{D}: \mathbb{Z}_{i k}^{2} \times \mathbb{C}^{*} \rightarrow \mathbb{N}_{+}
$$

Фиксируем ориентацию в плоскости однопараметрических тора $\mathbb{C}^{* 2}$. Фиксация этой ориентации задает параметризацию каждой одномерной орбиты на любой торической компактификации $M$ тора $\mathbb{C}^{* 2}$. Пусть вектор $\vec{n} \in \mathbb{Z}_{\mathrm{ir}}^{2}$ соответствует одномерной орбите $M_{\infty}^{\vec{n}}$ на поверхности $M$. В этом случае положим функцию $\operatorname{Mul}_{D}(\vec{n}, c)$ равной 
кратности, с которой точка с параметром $c$ на одномерной орбите $M_{\infty}^{\vec{n}}$ входит в дивизор $D$. Если вектор $\vec{n} \in \mathbb{Z}_{\mathrm{ir}}^{2}$ соответствует нульмерной орбите на поверхности $M$, то положим функщию $\operatorname{Mul}_{D}(\vec{n}, c)$ равной нулю для всех точек $c \in \mathbb{C}^{*}$.

ТЕОРемА. Дивизор D является дивизором пересечения некоторой кривой, не проходящей через нульмерные орбиты торической поверхности $M$, с обвединением $M_{\infty}$ одномерных орбит поверхности $M$, если и только если эта кривая является замыканием кривой, определенной в $\mathbb{C}^{* 2}$ уравнением $P=0$, причем функuия $\mathrm{Mul}_{P}$ полинома Лорана $P$ равна функиии $\mathrm{Mul}_{D}$ дивизора $D: \mathrm{Mul}_{P}=\mathrm{Mul}_{D}$.

ДокАЗАтЕльство. Торическая поверхность $M$ содержит тор $\mathbb{C}^{* 2}$. Всякая алгебраическая кривая, лежащая на поверхности $M$ и не содержащая одномерных орбит в качестве компонент, является замьканием кривой, лежашей в торе $\mathbb{C}^{2 *}$. Каждая кривая в $\mathbb{C}^{2 *}$ задается некоторьм уравнением $P=0$, где $P$ - полином Лорана. Рассмотрим функцию $\mathrm{Mul}_{P}$, построенную по этому полиному Лорана. Если вектор $\vec{n} \in \mathbb{Z}_{\mathrm{ir}}^{2}$ в веере поверхности $M$ соответствует нульмерной орбите $A$, то функция $\operatorname{Mul}_{P}(\vec{n}, \cdot)$ должна тождественно обрашаться в нуль на $\mathbb{C}^{*}$. В противном случае, как видно из теоремы $1^{\prime}$ о многоугольниках Ньютона, замыкание в поверхности $M$ кривой $P=0$ будет содержать нульмерную орбиту $A$. Пусть вектор $\vec{n} \in \mathbb{Z}_{\mathrm{ir}}^{2}$ в веере поверхности $M$ соответствует одномерной орбите $M_{\infty}^{\vec{n}}$. Тогда согласно теореме $1^{\prime}$ о многоугольниках Ньютона и утверждению 2 из $\S 3$, замькание кривой $P=0$ пересекает орбиту $M_{\infty}^{\vec{n}}$ только в точках, параметры которых являются корнями полинома $P_{\vec{n}}$. При этом кратность точки пересечения равна кратности соответствуюшего корня полинома $P_{\vec{n}}$. Теорема доказана.

Подведем итог. Пусть носитель дивизора $D$ лежит не более чем на двух орбитах. Тогда искомая кривая сушествует, если и только если

1) этих орбит ровно две и им соответствуют противоположные векторы $\vec{n}_{1}$ и $\vec{n}_{2}$, $\vec{n}_{1}+\vec{n}_{2}=0$

2) точка на орбите $M_{\infty}^{\vec{n}_{1}}$ с параметром $c$ входит в дивизор $D$ с той же кратностью, с которой в него входит точка с параметром $c^{-1}$ на орбите $M_{\infty}^{\vec{n}_{2}}$.

Пусть дивизор $D$ содержится в объединении не менее чем трех одномерных орбит. Тогда искомая кривая существует, если и только если вьполнены

1) условие Паскаля $\sum \operatorname{Mul}_{D}(\vec{n}, c)=0$;

2) условие Виета $\prod(-c)^{\operatorname{Mul}_{D}(\vec{n}, c)}=1$.

Если условия 1)-2) выполнены, то искомая кривая Г задается следующим образом. По функции $\mathrm{Mul}_{D}$ строится многоугольник Ньютона $\Delta_{D}$, для которого

$$
\operatorname{Lengh}_{\Delta_{D}}(\vec{n})=\sum_{c} \operatorname{Mul}_{D}(\vec{n}, c)
$$

Такой многоугольник определен однозначно с точностью до параллельного переноса. Далее, у полинома Лорана $P$ выделяются коэффициенты при всех мономах, соответствующих граничным точкам многоугольника $\Delta$. Эти коэффициенты определяются однозначно, с точностью до общего множителя, из условия, что в точке $c$ порядок полинома $P_{\vec{n}}$ равен $\operatorname{Mul}_{D}(\vec{n}, c)$. Все остальные коэффициенты полинома Лорана $P$ определяются произвольным образом. 
Обозначим через $\mathscr{R}(D)$ пространство всех кривых на поверхности $M$, не проходяших через нульмерные орбиты и пересекающихся с объединением одномерных орбит по дивизору $D$. Так как пропорциональные уравнения определяют одну и ту же кривую, $\mathscr{R}(D)$ является проективизацией пространства полиномов Лорана $P$, для кото$\operatorname{pux} \operatorname{Mul}_{P}=\operatorname{Mul}_{D}$.

Пространство $\mathrm{Mul}_{P}$ представляет собой дополнение к точке 0 в линейном пространстве размерности $(B(\Delta)+1)$, где $B(\Delta)$ - число внутренних целых точек многоугольника $\Delta, \Delta_{D}=\Delta(P)$. (Мы отождествляем полиномы Лорана, частное которых является мономом.) Поэтому пространство $\mathscr{R}(D)$ представляет собой проективное пространство размерности $B(\Delta)$.

\section{$\S 5$. Многоугольники без внутренних целых точек}

Начнем со списка исключительных многоугольников, ни один из которых не содержит внутри себя целых точек.

Список исключительных многоугольников.

1. Отрезок целочисленной длины $m$. Унимодулярньм преобразованием переводится в отрезок с вершинами $(0,0),(m, 0)$.

2. Треугольник, стороны которого имеют целочисленную длину 2. Унимодулярньм преобразованием переводится в симплекс с вершинами $(0,0),(2,0),(2,0)$.

3. Треугольник целочисленной высоты 1 с целочисленной длиной основания, равной $m$. Унимодулярньм преобразованием переводится в симплекс с вершинами $(0,0),(m, 0),(1,0)$.

4. Трапеция целочисленной высоты 1 с целочисленными длинами оснований $k$, $m$, где $0<k \leqslant m$. Целочисленным преобразованием переводится в трапецию с вершинами $(0,0),(m, 0),(1,0),(1, k)$.

Теорема. Если иелочисленный многоугольник содержит внутренние иелые точки, то для каждой его стороны l существует внутренняя целая точка, челочисленная высота которой относительно стороны l равна 1. Все челочисленные многоугольники без внутренних целых точек содержатся в списке исключительных многоугольников.

ДокАЗАТЕльство. Мы покажем, что если целочисленньй многоугольник не содержится в списке исключительных многоугольников, то для каждой его стороны $l$ найдется внутренняя целая точка, целочисленная высота которой относительно стороны $l$ равна 1. Отсюда, разумеется, вытекают оба утверждения теоремы. Унимодулярным преобразованием плоскости $(u, v)$ расположим многоугольник $\Delta$ так, чтобы он лежал в верхней полуплоскости $v \geqslant 0$, а сторона $l$ лежала на оси $v=0$. Если пересечение многоугольника $\Delta$ с прямой $v=1$ пусто, то целочисленньй многоугольник $\Delta$ - отрезок. Если это пересечение состоит из точки, то $\Delta$ - треугольник целочисленной высоты 1. Если отрезок $\Delta \cap(v=1)$ содержит внутреннюю целую точку, то либо эта точка является вну тренней точкой многоугольника $\Delta$, целочисленная высота которой относительно стороны $l$ равна 1 , либо отрезок $\Delta \cap(v=1)$ яВляется стороной многоугольника $\Delta$. В последнем случае многоугольник $\Delta$ является целочисленной трапецией с целочисленной высотой, равной 1 . Покажем, что если отрезок $\Delta \cap(v=1)$ 
не содержит внутренней целой точки, то многоугольник $\Delta$ содержится в списке исключительных многоугольников. Действительно, в этом случае отрезок $\Delta \cap(v=1)$ переводится в полосу $0 \leqslant u \leqslant 1$ унимодулярньм преобразованием плоскости, оставляюшим неподвижными все точки горизонтальной оси координат и переводяшим в себя все горизонтальные прямые.

Пусть отрезок $\Delta \cap(v=1)$ совпадает с отрезком $(0 \leqslant u \leqslant 1, v=1)$. Здесь возникают следуюшие случаи. Если сторона $l$ имеет длину 1 , то многоугольник $\Delta$ лежит в полосе $0 \leqslant u \leqslant 1$ и является трапецией с целочисленной высотой, равной 1 . Если сторона $l$ имеет длину 2 , то многогранник $\Delta$ является симплексом $(u \geqslant 0, v \geqslant 0$, $u+v \leqslant 2$ ) со сторонами целочисленной длины, равной 2 . В рассматриваемом случае не сушествует целочисленных многоугольников $\Delta$ со стороной $l$ длины больше 2 .

Если длина отрезка $\Delta \cap(v=1)$ меньше 1 , то встречаются лишь случаи, в которых длина стороны $l$ равна 1 , а многоугольник $\Delta$ является треугольником целочисленной высоты, равной 1 (с вершиной, расположенной либо на прямой $u=0$, либо на прямой $u=1)$. Теорема доказана.

\section{$\S$ 6. Многоугольник $\Delta_{D}$ с внутренней целой точкой}

В этом параграфе описьвается общая кривая из пространства $\mathscr{R}(D)($ см. $\S 4)$ в том случае, когда многоугольник $\Delta_{D}$ содержит внутреннюю целую точку.

Лемма. Пусть точка а лежит на одномерной орбите $M_{\infty}^{\vec{n}}$ поверхности $M$ и входит в дивизор $D$ с кратностью $k(a)$. Пусть дивизор $D$ допустим и многоугольник $\Delta_{D}$ содержит внутреннюю челую точку. Тогда общая кривая Г из пространства $\mathscr{R}(D)$ около точки а является гладкой, причем кривая Г касается орбиты $M_{\infty}^{\vec{n}}$ с кратностью касания $k(a)$.

ДокАЗАТЕльСтво. Пусть $c$ - параметр точки $а$ на орбите $M^{\vec{n}}$, и пусть $P=0-$ уравнение кривой в $\mathbb{C}^{* 2}$, где $\mathrm{Mul}_{P}=\mathrm{Mul}_{D}$.

Сделаем унимодулярное степенное преобразование тора $\mathbb{C}^{* 2}$, переводящее вектор $\vec{n}$ в вектор $\vec{e}_{1}$. После такого преобразования и сокращения уравнения на подходящий моном уравнение $P=0$ будет иметь следующие свойства:

1) полином Лорана $P$ будет регулярен в плоскости $(x, y)$ вне оси $(y=0)$;

2 ) его ограничение $P(0, y)$ на ось $(x=0)$ будет совпадать с полиномом $P_{\vec{n}}(y)$.

В координатах $(x, y)$ ось $(x=0)$ совпадает с орбитой $M_{\infty}^{\vec{n}}$, а точка $a-$ с точкой $(0, c)$. Если полином $P_{\vec{n}}$ имеет в точке $c$ корень кратности 1 , то лемма вытекает из теоремы о неявной функции. Пусть $c-$ корень полинома $P_{\vec{n}}$ кратности $\geqslant 2$. Согласно теореме из $\S 5$, внутри многоугольника Ньютона $\Delta$ находится целая точка с координатами $(1, k)$, где $k$ - некоторое целое число.

Поэтому при любых значениях параметра $\lambda$ полином $P_{\lambda}=P+\lambda x y^{k}$ лежит в рассматриваемом пространстве, т.е. $\operatorname{Mul}_{P_{\lambda}}=\operatorname{Mul}_{D}$. Если $\lambda \neq-c^{-k} \frac{\partial P}{\partial y}(0, c)$, то к уравнению $P_{\lambda}=0$ около точки $(0, c)$ применима теорема о неявной функции. Лемма доказана.

Теорема. Пусть дивизор D допустим, а его многоугольник Ньютона $\Delta$ содержит внутреннюю целую точку. Тогда общая кривая из пространства $\mathscr{R}(D)$ 
является гладкой неприводимой кривой рода $g=B(\Delta)$, где $B(\Delta)$ - число внутренних точек многоугольника $\Delta$. Пересечение общей кривой из пространства $\mathscr{R}(\Delta)$ с тором $\mathbb{C}^{* 2}$ является сферой с g ручками, из которой выколото $q$ точек, где $q$ - число геометрически различных точек в носителе дивизора $D$.

ДоказАтельство. Общее уравнение $P=0$, где $\mathrm{Mul}_{P}=\mathrm{Mul}_{D}$, задает неособую кривую в торе $\mathbb{C}^{* 2}$. Действительно, пусть точка $(k, m)$ - внутренняя для многоугольника $\Delta$. Перепишем уравнение $P(x, y)=0$ в виде $\widetilde{P} x^{-k} y^{-m}=\lambda$, где $\widetilde{P}=P-$ $\lambda x^{k} y^{m}$. По теореме Сарда-Бертини для почти всех значений параметра $\lambda$ кривая $\widetilde{P} x^{-k} y^{-m}=\lambda$ будет неособой. Согласно лемме общая кривая из пространства $\mathscr{R}(D)$ будет неособой и в точках пересечения с одномерными орбитами поверхности $M$. Поэтому общая кривая из пространства $\mathscr{R}(D)$ вообще не имеет особых точек. По теореме о неявной функщии малое изменение коэффищиентов уравнения гладкой кривой не меняет топологию этой кривой. Немного меняя коэффициенты при всех мономах, можно добиться, чтобы уравнение стало $\Delta$-невырожденньм. Применяя хорошо известные результаты о $\Delta$-невырожденных кривых [5], получим, что общая кривая $\mathscr{R}(D)$ будет неприводимой и что ее род равен $B(\Delta)$. Число геометрически различных точек пересечения гладкой кривой из пространства $\mathscr{R}(D)$ с объединением одномерных орбит равно числу геометрически различных точек в дивизоре $D$.

\section{$\S$ 7. Многоугольник $\Delta_{D}$ без внутренних целых точек}

Пусть дивизор $D$ на объединении одномерных орбит торической поверхности $M$ допустим и его многоугольник Ньютона $\Delta_{D}$ не содержит внутренних цельх точек. Полное описание этого случая основано на полном перечислении многоугольников без внутренних целых точек (см. $\S 5)$. Если $\Delta(D)$ не имеет внутренних целых точек, то сушествует с точностью до множителя лиш один полином Лорана $P$ такой, что $\operatorname{Mul}_{P}=\operatorname{Mul}_{\Delta}$, и одна единственная кривая в пространстве $\mathscr{R}(D)$. Будем обозначать эту кривую $r(D)$.

ТЕОРЕМа 1. При помощи степенной унимодулярной замены координат и умножения на моном полином Лорана $P$ можсно привести либо к многочлену степени $m$ с ненулевым свободным членом, не зависящему от первой координатной функиии, либо к квадратному полиному с ненулевым свободным членом и с ненулевыми коэффициентами при $x^{2}$ и при $y^{2}$, либо к полиному $y S_{k}(x)+Q_{m}(x)$, где $S_{k}$ и $Q_{m}$ - многочлены степеней $k u m, m>0, m \geqslant k \geqslant 0$, с ненулевыми свободными членами.

ДоКАЗАТЕЛЬСТВо ТЕОРЕМЫ немедленно вытекает из рассмотрения списка многоугольников без внутренних целых точек (см. $\S 5)$.

Предположим дополнительно, что уравнение $P=0$ неприводимо. Тогда после степенной замены координат в первом случае оно определяет горизонтальную прямую, во втором случае - гладкую квадрику, а в третьем случае - график рациональной функции. Мы видим, что неприводимая кривая $r(D)$ ведет себя в точности как общая кривая из пространства $\mathscr{R}(D)$ в случае, когда многоугольник $\Delta_{D}$ имеет внутренние целые точки. 
Теорема 2. Пусть дивизор D допустим и его многоугольник Ньютона $\Delta$ не содержит внутренних цельх точек. Пусть дополнительно единственная кривая $r(D)$ в пространстве $\mathscr{R}(D)$ неприводима. Тогда она является гладкой раичональной кривой на поверхности $M$. Если дивизор $D$ содержит точку а $c$ кратностью $k(a)$, то кривая $r(D)$ в точке а касается $c$ кратностью $k(a)$ с одномерной орбитой, проходящей через точку а.

Нам осталось описать в терминах дивизора $D$ условия приводимости кривой и посмотреть, что происходит в этом случае.

Случай отрезка. Пусть функция $\mathrm{Mul}_{D}$ дивизора $D$ имеет ровно два характеристических вектора $\vec{n}_{1}$ и $\vec{n}_{2}$ таких, что $\vec{n}_{1}+\vec{n}_{2}=0$, и обладает свойством возвратности $\operatorname{Mul}_{D}\left(\vec{n}_{1}, c\right)=\operatorname{Mul}_{D}\left(\vec{n}_{2}, c^{-1}\right)$.

Многоугольник Ньютона $\Delta_{D}$ в этом случае является отрезком. Для описания кривой $r(D)$ сделаем унимодулярньй автоморфизм тора $\mathbb{C}^{* 2} \subset M$, переводяший вектор $\vec{n}_{1}$ из алгебры Ли тора $\mathbb{C}^{* 2}$ в вектор $\vec{e}_{1}$, где $\vec{e}_{1}=(1,0)$. После такого преобразования кривая $r(D)$ в $\mathbb{C}^{* 2}$ с координатами $(x, y)$ будет состоять из объединения горизонтальных прямых $y=c_{i}$, причем прямая $y=c_{i}$ входит в кривую $r(D)$ с той же кратностью, с которой точка с параметром $c_{i}$ на орбите $M^{\vec{n}_{1}}$ входит в дивизор $D$.

В частности, кривая $r(D)$ не содержит кратных компонент, если и только если функиия $\mathrm{Mul}_{D}$ принимает лишь значения 0 и 1 . Кривая $r(D)$ некратна $и$ неприводима, если и только если функиия $\mathrm{Mul}_{D}$ равна 1 ровно в двух точках $\left(\vec{n}_{1}, c\right)$ u $\left(-\vec{n}_{1}, c^{-1}\right)$ и равна нулю во всех остальных точках.

Переходим к случаю двумерных многоугольников $\Delta_{D}$. Допустимые дивизоры $D$ в этом случае удовлетворяют условию Виета. Комбинаторные типы многоугольников $\Delta$ в рассматриваемых ниже случаях различаются, и условие Паскаля принимает разные формы.

Случай симплекса со сторонами целочисленной длины 2. В рассматриваемом случае дивизор $D$ содержит по 2 точки (с учетом кратности) на трех орбитах, соответствуюших векторам $\vec{n}_{1}, \vec{n}_{2}, \vec{n}_{3}$. Условие Паскаля принимает следуюший вид:

$$
\vec{n}_{1}+\vec{n}_{2}+\vec{n}_{3}=0 .
$$

УТВЕРЖДЕНИЕ 1. Кривая $r(D)$ приводима, если и только если в носителе дивизора $D$ можно выбрать по одной точке на каждой из трех орбит так, чтобь произведение их параметров равнялось (-1). В этом случае кривая состоит из двух компонент. Эти компоненты сливаются в кратную компоненту, если и только если все точки дивизора дополнительно являются кратными. Если же эти компоненты являются различными, то они пересекаются ровно в одной точке поверхности $M$. Эта точка не принадлежсит тору $\mathbb{C}^{* 2}$, если и только если одна из точек дивизора $D$ является кратной.

ДокАЗАТЕльство. Степенным преобразованием кривая $r(D)$ переводится в квадрику. Для квадрики утверждение очевидно.

ЗАмЕчАнИЕ. Если допустимый дивизор содержит три двукратных точки, то по условию Виета $\left[\left(-\xi_{1}\right)\left(-\xi_{2}\right)\left(-\xi_{3}\right)\right]^{2}=1$, где $\xi_{i}$ - параметры этих точек. Возникает два случая.

1) случай $\left(-\xi_{1}\right)\left(-\xi_{2}\right)\left(-\xi_{3}\right)=1$ соответствует одной компоненте кратности два; 
2) случай $\left(-\xi_{1}\right)\left(-\xi_{2}\right)\left(-\xi_{3}\right)=-1$ соответствует неособой кривой; степенньм преобразованием такую кривую можно перевести в параболу, касающуюся осей координат.

Случай треугольника целочисленной высоты 1. Пусть дивизор $D$ содержит точки на трех орбитах, соответствуюших векторам $\vec{n}_{1}, \vec{n}_{2}, \vec{n}_{3}$. Пусть на орбите, соответствуюшей вектору $\vec{n}_{1}$, дивизор $D$ содержит не меньше точек, чем на любой из оставшихся орбит. Пусть это число точек равняется числу $k \geqslant 1$. Рассматриваемый случай определяется следующими условиями:

1) на каждой из двух оставшихся орбит дивизор имеет ровно по одной точке;

2) справедливы соотношения $k \vec{n}_{1}+\vec{n}_{2}+\vec{n}_{3}=0,\left|\operatorname{det}\left(\vec{n}_{1}, \vec{n}_{2}\right)\right|=\left|\operatorname{det}\left(\vec{n}_{1}, \vec{n}_{3}\right)\right|=1$.

При вьполнении условий 1$)-2$ ) условие допустимости дивизора $D$ состоит в вьполнении условия Виета.

УТВЕРЖДЕНИЕ 2. При выполнении перечисленных условий кривая $r(D)$ всегда будет неприводимой.

ДокАЗАТЕльство. Треугольник с целочисленной высотой 1 не раскладывается в сумму Минковского целочисленных многоугольников. Полином Лорана с таким многоугольником Ньютона неприводим.

Случай трапеции целочисленной высоты 1. Этот случай появляется в следуюших обстоятельствах: дивизор $D$ содержит точки на четырех орбитах, соответствующих векторам $\vec{n}_{1}, \vec{n}_{2}, \vec{n}_{3}, \vec{n}_{4}$. На одной из орбит он содержит не менњше точек, чем на других орбитах. Пусть эта орбита соответствует вектору $\vec{n}_{1}$. Обозначим число точек на ней через $m, m \geqslant 1$. Среди векторов $\vec{n}_{2}, \vec{n}_{3}, \vec{n}_{4}$ есть вектор $-\vec{n}_{1}$. Будем считать, что это вектор $\vec{n}_{3}$. Дивизор должен иметь ровно по одной точке на орбитах, соответствующих векторам $\vec{n}_{2}$ и $\vec{n}_{4}$, и некоторое число $k, k \geqslant 1$, точек на орбите, соответствуюшей вектору $-\vec{n}_{1}=\vec{n}_{3}$. Должны выполняться соотношение Паскаля $m \vec{n}_{1}+\vec{n}_{2}+k \vec{n}_{3}+\vec{n}_{4}=0$ и соотношение $\left|\operatorname{det}\left(\vec{n}_{1}, \vec{n}_{2}\right)\right|=\left|\operatorname{det}\left(\vec{n}_{1}, \vec{n}_{3}\right)\right|=1$.

Приведенные дискретные условия эквивалентны условию Паскаля существования трапеции $\Delta_{D}$ и требованию, чтобы трапеция $\Delta_{D}$ не содержала внутренних целых точек. При вьполнении этих условий дивизор $D$ будет допустим, если и только если вьполнено соотношение Виета.

Разложим теперь дивизор $D$ в линейную комбинацию с неотрицательными целыми коэффициентами некоторых эффективных дивизоров $D_{i}$. Пусть $c_{1}, \ldots, c_{j}$ - параметры геометрически различных точек в носителе дивизора $D$ на орбите, соответствующей вектору $\vec{n}_{1}$. С каждым номером $i, 1 \leqslant i \leqslant j$, свяжем следуюший двуточечньй дивизор $D_{i}$ : дивизор содержит точку $A_{i}$ с параметром $c_{i}$ на орбите $M_{\infty}^{\vec{n}_{1}}$ и точку $B_{i}$ с параметром $c_{i}^{-1}$ на орбите $M_{\infty}^{-\vec{n}_{1}}$. Положим $k(i)$ равным минимуму из кратностей вхождения в дивизор $D$ точек $A_{i}$ и $B_{i}$. Положим $D_{\nu}=\sum k(i) D_{i}$ и $D_{0}=D-D_{\nu}$.

УТВЕРЖДЕНИЕ 3. Описанное выше разложсение дивизора $D$ в сумму $D=D_{0}+$ $\sum k(i) D_{i}$ соответствует разложению кривой $r(D)$ на неприводимье компонен$m b l$ :

$$
r(D)=r\left(D_{0}\right)+\sum_{i=1} k(i) r\left(D_{i}\right)
$$


Каждая из кривых $r\left(D_{i}\right)$ является гладкой рациональной кривой на торической поверхности $M$. Кривая $r(D)$ будет неприводима, если и только если все числа $k(i)$ равны 0. Кривая $r(D)$ не содержит кратных компонент, если и только если все числа $k(i)$ меньше 2. При $i>0$ различные кривые $r\left(D_{i}\right)$ на торической поверхности не пересекаются друг с другом. Каждая из кривых $r\left(D_{i}\right)$ nри $i>0$ пересекается с кривой $r\left(D_{0}\right)$ на поверхности $M$ ровно по одной точке. Эта единственная точка пересечения не лежит в торе $\mathbb{C}^{* 2}$, если и только если носители дивизоров $D_{0}$ и $D_{i}$ пересекаются.

ДОКАЗАТЕЛЬСТВО УТВЕРЖДЕНИЯ сводится к следующему. Полином $y S_{k}(x)+$ $Q_{m}(x)$ приводим, если и только если он делится на некоторьй полином $G(x)$. Компоненты приведенного вьше разложения соответствуют корням $c_{i}$ полинома $G(x)$, числа $k_{i}$ - кратностям этих корней. Кривые $r\left(D_{i}\right)$ соответствуют вертикальным линиям $x=c_{i}$, а кривая $r\left(D_{0}\right)$ - графику рациональной функции $y=S_{k}(x) / Q_{m}(x)$ (числитель и знаменатель дроби надо сократить на общий множитель $G(x))$.

\section{§ 8. Мероморфные векторные функции на компактных кривых}

Рассмотрим тройку $\Gamma, f, g$, состояшую из компактной (не обязательно связной) кривой Г и мероморфной вектор-функции $(f, g)$ на ней. Мы всегда будем предполагать, что ни на какой компоненте связности кривой $\Gamma$

1) ни одна из функций $f, g$ не обрашается в тождественньй нуль,

2) вектор-функция $(f, g)$ не является постоянной.

$\mathrm{C}$ каждой тройкой $\Gamma, f, g$ свяжем функцию $\mathrm{Mul}_{\Gamma f g}$ на произведении $\mathbb{Z}_{\mathrm{ir}}^{2} \times \mathbb{C}^{*}$, сопоставляющую несократимому вектору $\vec{n}$ и ненулевому комплексному числу с сумму кратностей ростков во всех точках на кривой $\Gamma$, в которых росток вектор-функции имеет тип $\vec{n}$, а приведенное число Вейля этого ростка равно $c$ (см. $\S 2)$. Другими словами,

$$
\operatorname{Mul}_{\Gamma f g}(\vec{n}, c)=\sum_{a} k(a)
$$

где суммирование ведется по всем точкам $a$, в которых тип ростка $(f, g)$ равен $\vec{n}$ и $[f, g]_{a}=c$.

Настояший параграф̆ посвяшен решению следуюших задач 1-3.

ЗАДАчА 1. Дана функиия Mul на $\mathbb{Z}_{\mathrm{ir}}^{2} \times \mathbb{C}^{*}$, принимающая иелье неотрицательные значения и равная нулю всюду кроме конечного числа точек. Найти тройки $\Gamma, f, g$, для которьх $\operatorname{Mul}_{\Gamma f g}$ равна Mul.

Решения задачи 1 делятся на кратные и некратные. Скажем, что тройка $\Gamma, f, g$ является некратной, если вектор-функция $(f, g)$ склеивает не более чем конечное число точек на кривой Г.

Задача 1 имеет следующие варианты.

ЗАДАчА 2. Найти некратные решения задачи 1.

ЗАДАчА 3. Найти некратные решения $\Gamma, f, g$ задачи 1, для которых кривая Г связна. 
Лемма 1. Пусть $\pi:(\Upsilon, b) \rightarrow(\Gamma, a)-$ росток $l$-листного отображсения кривой $\Upsilon$ в кривую $Г и(f, g)$ - росток мероморфной вектор-функиии в точке а $\in$ г. Тогда росток $\left(\pi^{*} f, \pi^{*} g\right)$ мероморфной вектор-функиии в точке $b \in \Upsilon$ имеет тот же тип, что и росток $(f, g)$, а его кратность в $l$ раз больше, чем кратность ростка $(f, g)$. Справедливы равенства:

1) $\left[\pi^{*} f, \pi^{*} g\right]_{b}=[f, g]_{a}$

2) $\left\{\pi^{*} f, \pi^{*} g\right\}_{b}=\{f, g\}_{a}^{l}$.

ДокАЗАТЕЛЬСТВо. Можно так выбрать локальные параметры $u$ и $v$ на ростках кривых Г и $\Upsilon$, что отображение $\pi$ будет задаваться формулой $\pi(v)=v^{l}=u$. При этом если $f=c_{1} u^{b_{1}}+\cdots, g=c_{2} u^{b_{2}}+\cdots$, то $\pi^{*} f=c_{1} v^{l b_{1}}+\cdots, \pi^{*} g=c_{2} v^{l b_{2}}+\cdots$. Откуда и вытекают нужные равенства.

Пусть кривая Г состоит из компонент связности $\Gamma_{1}, \ldots, \Gamma_{k}$. С каждой тройкой $\Gamma, f, g$ связано $k$ троек $\Gamma_{1}, f, g ; \ldots ; \Gamma_{k}, f, g$. (Мы обозначаем одним и тем же символом функцию на кривой $Г$ и ограничение этой функции на компоненту связности $\Gamma_{i}$ этой кривой.) Пусть $\pi: \Upsilon \rightarrow \Gamma$ - разветвленное накрытие кривой $\Upsilon$ над кривой $\Gamma$, при котором число точек в прообразе $\pi^{-1}(a)$ общей точки $a$ на компоненте $\Gamma_{i}$ равно $\mu_{i}($ мы не предполагаем, что полньй прообраз $\pi^{-1}\left(\Gamma_{i}\right)$ компоненты $\Gamma_{i}$ связен).

ЛЕмма 2. Справедливо равенство:

$$
\operatorname{Mul}_{\Upsilon F G}=\sum \mu_{i} \operatorname{Mul}_{\Gamma_{i} f g}
$$

əде $F=\pi^{*} f u G=\pi^{*} g$.

Лемма 2 немедленно вытекает из леммы 1.

Тройке $\Upsilon, F, G$ сопоставим плоскую кривую $\Gamma^{\text {gеот }}$, являющуюся замыканием образа кривой $\Upsilon$ в торе $\mathbb{C}^{* 2}$ при мероморфном отображении, переводящем точку $a$ в точку $(F(a), G(a))$.

Для каждой неприводимой компоненты этой кривой определена ее кратность $\mu_{i}$, равная числу прообразов у общей точки на рассматриваемой компоненте при отображении $(F, G): \Upsilon \rightarrow \mathbb{C}^{* 2}$.

Плоскую алгебраическую кривую $\Gamma^{\mathrm{alg}}$, геометрически совпадаюшую с кривой $\Gamma^{\mathrm{geom}}$, но в которой каждая ее компонента рассматривается с кратностью $\mu_{i}$, назовем характеристической плоской кривой для тройки $\Upsilon, F, G$.

Полином Лорана $P(x, y)=\prod P_{i}^{\mu_{i}}(x, y)$, где $P_{i}(x, y)$ - неприводимьй полином Лорана, равньй нулю на $i$-й компоненте, и $\mu_{i}$ - кратность этой компоненты, назовем $x a$ рактеристическим полиномом Лорана для тройки $\Upsilon, F, G$. Характеристический полином определен с точностью до умножения на ненулевую константу (мы отождествляем два полинома Лорана, частное которых является мономом). Кривая $\Gamma^{\mathrm{alg}}$ задается уравнением $P=0$.

Нормализация $\bar{\Gamma}$ характеристической кривой $\Gamma^{\text {geom }}$ состоит из компонент $\bar{\Gamma}_{i}$, являюшихся нормализациями компонент $\Gamma_{i}^{\text {geom }}$ характеристической кривой. На нормализации $\bar{\Gamma}$ определена мероморфная вектор-функция $(x, y): \bar{\Gamma} \rightarrow \mathbb{C}^{* 2}$, задаюшая ее бирациональньй изоморфизм с кривой $\Gamma^{\mathrm{geom}}$. 
ТеОРема 1. Пусть $\pi: \Upsilon \rightarrow \bar{\Gamma}$ - разветвленное накрытие над нормализацией кривой $\Gamma^{\text {geom }}$ такое, что число прообразов общей точки на компоненте $\bar{\Gamma}_{i}$ равно кратности $\mu_{i}$ әтой компоненты. Тогда характеристическая кривая для тройки $\Upsilon, F, G$, где $F=\pi^{*} x$ u $G=\pi^{*} y$, совпадает с кривой $\Gamma^{\mathrm{alg}}$. Обратно, если характеристическая кривая для тройки $\Upsilon, F, G$ совпадает с кривой $\Gamma^{\mathrm{alg}}$, то существует разветвленное накрытие $\pi: \Upsilon \rightarrow \bar{\Gamma}$ такое, что $F=\pi^{*} x, G=\pi^{*} y$, и число прообразов общей точки на компоненте $\bar{\Gamma}_{i}$ равно кратности $\mu_{i}$ компоненты $\Gamma_{i}^{\text {geom }}$ в $\Gamma^{\text {geom }}$.

Теорема 1 непосредственно вытекает из определений.

СлЕДСТВИЕ 1. Если характеристическая кривая не имеет кратных компонент, то тройка $\Upsilon, F, G$ однозначно с точностью до изоморфизма определяется характеристической кривой. Ею является нормализачия характеристической кривой вместе с вектор-функцией $(x, y)$ на ней.

Следствие 1 вытекает из теоремы 1 , так как в рассматриваемом случае разветвленное накрытие является изоморфизмом.

СлЕДСТВИЕ 2. Если характеристическая кривая имеет кратные компоненты, то тройка $\Upsilon, F, G$ однозначно восстанавливается после фиксации следующих данных: набора любых конечных множсеств $A_{i}$ на неприводимых компонентах $\bar{\Gamma}_{i}$ нормализачии характеристической кривой, имеющих кратности $\mu_{i} \geqslant 2$, и гомоморфизмов фундаментальных групп $\pi_{1}\left(\bar{\Gamma}_{i} \backslash A_{i}\right)$ в группь перестановок $\mu_{i}$ әлементов.

Следствие 2 вытекает из теоремы 1 и классификации разветвленных накрытий. Следствие 2 показьвает, что сушествуют многопараметрические семейства различных троек $\Upsilon, F, G$ с заданной характеристической кривой, имеющей кратные компоненты. Непрерьвными параметрами являются множества точек ветвления $A_{i}$ на кратных компонентах, которые можно выбирать произвольно (и которые могут содержать любое число точек).

ТЕОРема 2. Для всякой тройки $\Upsilon, F, G$ справедливо равенство

$$
\operatorname{Mul}_{\Upsilon F G}=\operatorname{Mul}_{P}
$$

где $P$ - характеристический полином Лорана тройки $\Upsilon, F, G$.

ДоКАЗАТЕЛЬСТво. Достаточно воспользоваться теоремой $1^{\prime}$ о многоугольниках Ньютона для уравнения $P=0$ характеристической кривой тройки $\Upsilon, F, G$ и теоремой 1 из этого параграфа.

Мы имеем теперь полное решение задачи 1.

Действительно, мы знаем условия на функцию Mul, необходимые и достаточные для того, чтобы она являлась функцией $\mathrm{Mul}_{P}$ некоторого полинома Лорана $P$. С каждым решением $P$ задачи $\mathrm{Mul}_{P}=\mathrm{Mul}$ связаны решения задачи 1 , для которых кривая $P=0$ в $\mathbb{C}^{* 2}$ является характеристической. Если кривая $P=0$ не имеет кратных компонент, с ней связана ровно одна с точностью до изоморфизма тройка, решаюшая задачу 1. Именно, такой тройкой является нормализация характеристической кривой вместе с вектор-функцией $(x, y)$ на ней (см. следствие 1$)$. Если же кривая $P=0$ 
содержит кратные компоненты, с ней связано много решений задачи 1, но все они являются кратными. Каждая тройка с характеристической кривой $P=0$ получается из накрытия над характеристической кривой. Множество таких накрытий содержит бесконечное число компонент растущей размерности (см. следствие 2).

Перейдем теперь к задачам 2 и 3 . Согласно теоремам 1 и 2 , решение задачи 2 (задачи 3) связано с полиномами Лорана $P$ без кратных множителей (с неприводимыми полиномами Лорана $P$ ) такими, что $\mathrm{Mul}_{P}=\mathrm{Mul}$. Решения задачи 2 (задачи 3 ) доставляют нормализации кривых $P=0$ вместе с вектор-функциями $(x, y)$ на них.

Остановимся на возникающих здесь случаях.

1. Случай, когда $\Delta(\mathrm{Mul})$ содержит внутреннюю целую точку.

Пусть для функции $\mathrm{Mul}$ вьполнены условия существования полинома Лорана $P$ такого, что $\mathrm{Mul}_{P}=\mathrm{Mul}$, причем многоугольник $\Delta(\mathrm{Mul})$ содержит внутреннюю целую точку. Тогда для общего полинома Лорана $P$ такого, что $\mathrm{Mul}_{P}=\mathrm{Mul}$, кривая $P=0$ в $\mathbb{C}^{* 2}$ будет связной и неособой кривой (см. $\left.\S 6\right)$. Поэтому в этом случае из разрешимости задачи 1 следует разрешимость как задачи 2 , так и задачи 3 . Решения этих задач образуют комплексные многообразия размерностей $B(\Delta)$, где $B(\Delta)-$ число внутренних точек многоугольника $\Delta(\mathrm{Mul})$.

2. Случай, когда $\Delta(\mathrm{Mul})$ не содержит внутренней целой точки.

Если задача нахождения полинома Лорана такого, что $\mathrm{Mul}_{P}=\mathrm{Mul}$, разрешима, то она имеет единственное с точностью до обратимого множителя решение. При этом если соответствуюший полином Лорана $P$ неприводим, то кривая $P=0$ вместе с вектор-функцией $(x, y)$ доставляет единственное решение задач $1-3$. Если соответствующий полином Лорана приводим, но не имеет кратных компонент, то кривая $P=0$ вместе с вектор-функцией $(x, y)$ доставляет единственное решение задач 1 и 2 . Задача 3 в этом случае неразрешима.

Если кривая $P=0$ имеет кратные компоненты, то задачи 2 и 3 неразрешимы (а задача 1 имеет бесконечное число решений).

По функции Mul легко узнать, с каким из случаев мы имеем дело (см. §7). Итак, приведены условие разрешимости задач 1,2 и 3 , решения этих задач полностью описаны.

\section{§. Уточнение теоремы Вейля}

Что означают с точки зрения теории функций на кривых условия на функцию Mul, необходимые и достаточные для того, чтобы она была функцией $\mathrm{Mul}_{\Gamma f g}$ ?

1. Условие Паскаля $\sum \operatorname{Mul}(\vec{n}, c) \vec{n}=0$. Условие Паскаля для функции $\mathrm{Mul}=$ $\operatorname{Mul}_{\Gamma f g}$ означает, что степень дивизора как мероморфной функции $f$, так и $g$ на компактной кривой $\Gamma$ равна нулю. С точки зрения геометрии кривых выполнение этого условия очевидно. Если оно вьполнено, то по функции Mul можно построить многоугольник $\Delta=\Delta(\mathrm{Mul})$.

2. Условие Виета $\sum(-c)^{\operatorname{Mul}(\vec{n}, c)}=1$. Условие Виета для функции $\mathrm{Mul}=\mathrm{Mul}_{\Gamma f g}$ совпадает со следующей теоремой Вейля.

ТЕОРема ВеЙля. Для любой компактной аналитической кривой Г и двух ме- 
роморфных функций $f, g$ на ней справедливо соотношение

$$
\prod_{a \in \Gamma}\{f, g\}_{a}=1
$$

Тем самым, мы, во-первых, доказали теорему Вейля. Во-вторых, мы показали, что при вьполнении дискретного условия Паскаля $\sum \operatorname{Mul}(\vec{n}, c)=0$ в том случае, если многоугольник $\Delta(\mathrm{Mul})$ не является отрезком, теорема Вейля доставляет единственное необходимое условие на существование кривой Г и вектор-функции $(f, g)$ таких, что $\mathrm{Mul}_{\Gamma f g}=\mathrm{Mul}$.

3. Условие возвратности $\operatorname{Mul}(\vec{n}, c)=\operatorname{Mul}\left(-\vec{n}, c^{-1}\right)$. Это условие с необходимостью должно вьполняться лишь в специальном случае, когда функция Mul имеет не более двух характеристических векторов $\vec{n}_{1}$ и $\vec{n}_{2}$. Мы уже имеем полное описание этого случая. Однако, так как, во-первых, в рассматриваемом специальном случае мы имеем уточнение теоремы Вейля, и так как, во-вторых, полный разбор этого случая с точки зрения теории функций на кривой $Г$ тоже очень прост, мы приведем его ниже.

Скажем, что тройка $\Gamma, f, g$ исключительная, если сушествует не более чем два ненулевых вектора, которые являются векторами типа ростка $(f, g)_{a}$ для какой-либо точки $a \in \Gamma$. Будем говорить, что вектор $\vec{n} \in \mathbb{Z}_{\mathrm{ir}}^{2}$ является характеристическим для функции $\operatorname{Mul}_{\Gamma f g}$, если существует число $c \in \mathbb{C}^{*}$ такое, что $\operatorname{Mul}_{\Gamma f g}(\vec{n}, c) \neq 0$.

Лемма 1. Для всякой исключительной тройки $\Gamma, f, g$ существует ровно два характеристических вектора, и их сумма равна нулю.

Эта лемма вытекает из условия Паскаля.

Покажем, что случай любой пары характеристических векторов $\vec{n}_{1}, \vec{n}_{2}, \vec{n}_{1}+\vec{n}_{2}=$ 0 , сводится к легко описьваемому случаю, когда один из них (скажем, вектор $\vec{n}_{1}$ ) равен вектору $\vec{e}_{1}=(1,0)$. Обозначим через $A$ унимодулярную матрицу такую, что $A \vec{e}_{1}=\vec{n}_{1}$.

ЛЕмма 2. 1) Тройка $\Gamma, f, g$ является исключительной тройкой с характеристическими векторами $\vec{n}_{1} u-\vec{n}_{1}$, если и только если тройка $\Gamma, F, G$, в которой вектор-функчия $(F, G)$, полученная степенным преобразованием с матрицей $A^{-1}$ из вектор-функиии $(f, g)$, имеет характеристические векторы $\vec{e}_{1} u-\vec{e}_{1}$.

2) Исключительная тройка $\Gamma, F, G$ имеет характеристические векторь $\vec{e}_{1}$, $-\vec{e}_{1}$, если и только если функция $G$ постоянна на каждой компоненте связности кривой $Г$.

ДокАЗАТЕЛЬСтво. Первое утверждение леммы 2 вытекает из леммы $2 \S 2$. Вектор-функция $(F, G)$ имеет характеристические векторы $\vec{e}_{1},-\vec{e}_{1}$, если и только если функция $G$ ни в одной точке кривой не обрашается в нуль и не имеет полюсов. Такая функция постоянна на каждой компоненте связности кривой Г. Лемма 2 доказана.

Теорема 1. Для всякой исключительной тройки $\Gamma, f, g$ функиия $\mathrm{Mul}=\mathrm{Mul}_{\Gamma f g}$ обладает свойством возвратности

$$
\operatorname{Mul}_{\Gamma f g}(\vec{n}, c)=\operatorname{Mul}_{\Gamma f g}\left(-\vec{n}, c^{-1}\right) .
$$


ДокАЗАТЕльСтво. 1) Пусть кривая Г связна и функция $g$ равна константе: $g \equiv c$. Тогда в нулях $a$ функции $f$ имеем $[f, c]_{a}=c$, поэтому функция $\operatorname{Mul}_{\Gamma f g}$ на паре $\left(\vec{e}_{1}, c\right)$ равна $\operatorname{deg} f$. В полюсах функции $f$ имеем $[f, c]_{c}=c^{-1}$, поэтому функция $\operatorname{Mul}_{\Gamma f g}$ на паре $\left(-\vec{e}_{1}, c^{-1}\right)$ равна $\operatorname{deg} f$. Во всех остальных точках функция $\operatorname{Mul}_{\Gamma f g}$ равна нулю.

2) Лемма 2 сводит общий случай к случаю, рассмотренному в пункте 1 ).

Tеорема 2. Пусть функиия Mul с характеристическими векторами $\vec{n}_{1},-\vec{n}_{1}$ обладает свойством возвратности и функиия $\varphi(c)=\operatorname{Mul}\left(\vec{n}_{1}, c\right)$ отлична от нуля в точках $c_{1}, \ldots, c_{m}$, причем $\varphi\left(c_{i}\right)=k_{i}$. Тройки $\Gamma, f, g$, для которых $\mathrm{Mul}_{\Gamma f g}=$ $\mathrm{Mul}$, находятся во взаимнооднозначном соответствии с наборами из $m$ раз-

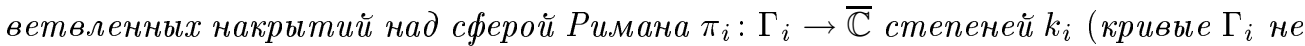
обязательно связны). При этом набору накрытий соответствует следующая тройка $\Gamma, f, g$. Пусть $\Gamma, F, G-$ тройка, в которой кривая $\Gamma=\cup \Gamma_{i}, a(F, G)-$ вектор-функиия, ограничение которой на кривую $\Gamma_{i}$ равно вектор-функции $\left(c_{i}, \pi_{i}\right)$. Тройка $\Gamma, f, g$ - степенное преобразование тройки $\Gamma, F, G$ с такой унимодулярной матрицей $A$, что $A \vec{e}_{1}=\vec{n}_{1}$.

Доказательство теоремы 2 вытекает из леммы 2 и вычисления функции $\mathrm{Mul}_{\Gamma f g}$ для тройки, в которой кривая $Г$ связна, а функция $g$ постоянна (см. п. 1 ) доказательства теоремы 1).

СлеДСТвиЕ. 1. Для всякой ненулевой исключительной функции Mul, обладающей свойством возвратности, существуют тройки $\Gamma, f, g$, для которых $\mathrm{Mul}_{\Gamma f g}=\mathrm{Mul}$.

2. При этом некратные тройки $\Gamma, f, g$ существуют, если и только если все ненулевье значения функции Mul равны 1. Для каждой такой функиии Mul существует ровно одна тройка $\Gamma, f, g$.

3. Некратная тройка $\Gamma, f, g$, в которой кривая Г связна, существует, если и только если функиия Mul отлична от нуля ровно в двух точках и равна в этих mочках 1.

Мы повторили разбор исключительного случая, не прибегая явно к многоугольникам Ньютона, а используя лишш простейшие свойства мероморфных функций на компактных кривых.

\section{$\S 10$. Теорема Абеля и соотношение Виета}

Пусть $D$ - дивизор, лежаший на объединении одномерных орбит $M_{\infty}$ торической поверхности $M$. Среди условий существования кривой, пересекающей $M_{\infty}$ по дивизоpy $D$, есть непрерьвное условие Виета (см. $\S 4$ ). Обычно непрерьвное условие такого рода возникает из теорем типа теоремы Абеля (см. [6]). В этом параграфе мы приводим простейший вариант теоремы Абеля, тесно связанный с соотношением Виета.

Пусть на компактной (не обязательно связной) кривой $\Lambda$ задан конечный набор $\left\{A_{i}\right\}$ непересекаюшихся конечных множеств $A_{1}, \ldots, A_{m}, A_{i} \cap A_{j}=\varnothing$.

Скажем, что мероморфная форма $\omega$ на кривой $\Lambda$ является $\left\{A_{i}\right\}$-регулярной, если

1) форма $\omega$ регулярна на дополнении кривой $\Lambda$ к множеству $\bigcup_{i=1}^{m} A_{i}$,

2) форма $\omega$ имеет полюса не вьше первого порядка в точках множества $\bigcup_{i=1}^{m} A_{i}$, 
3 д для каждого множества $A_{i}$ сумма вычетов формы $\omega$ по точкам множества $A_{i}$ равна 0.

Скажем, что мероморфная функция $\varphi$ на кривой $\Lambda$ является $\left\{A_{i}\right\}$-мероморфной, если функция $\varphi$ принимает одно и то же значение (возможно, равное $\infty$ ) во всех точках множества $A_{i}$, т.е. при $i=1, \ldots, m$, если $a, b \in A_{i}$, то $\varphi(a)=\varphi(b)$.

Теорема 1 (вариант теоремы Абеля). След $\left\{A_{i}\right\}$-регулярной формы $\omega$ nрu $\left\{A_{i}\right\}$-мероморфном отображении $\varphi: \Lambda \rightarrow \mathbb{C}$ тождественно равен нулю.

ДокАЗАТЕЛЬСтво. Начнем с локального вычисления. Пусть $\varphi(u)=u^{k}$ и $\omega=$

$a \frac{d u}{u}+\omega_{1}$, где $\omega_{1}-$ голоморфная форма. Тогда след формы $\omega$ имеет полюс первого порядка с тем же вьгетом, что и у формы $\omega$. Действительно, пусть $v=u^{k}, v^{1 / k}-$ ветвь корня, и $\varepsilon_{i}, i=1, \ldots, k,-$ корни $k$-й степени из 1. Тогда

$$
\operatorname{Tr} \omega=\operatorname{Tr} \omega_{1}+a \sum_{1 \leqslant i \leqslant k}\left(\frac{\frac{1}{k} \varepsilon_{i} v^{1 / k-1}}{\varepsilon_{i} v^{1 / k}}\right) d v=\operatorname{Tr} \omega_{1}+a \frac{d v}{v}
$$

По теореме Абеля след голоморфной формы $\omega_{1}$ голоморфен.

Из локального вычисления и обычной теоремы Абеля, во-первых, вытекает, что форма $\operatorname{Tr} \omega$ могла бы иметь лиш полюса не выше первого порядка и лишь в точках образа множества $\bigcup A_{i}$. Во-вторых, из локального вычисления и определения $\left\{A_{i}\right\}$-регулярной формы и $\left\{A_{i}\right\}$-мероморфного отображения вытекает, что форма $\operatorname{Tr} \omega$ вообше не имеет полюсов. Следовательно, $\operatorname{Tr} \omega \equiv 0$.

Как и в обычной теореме Абеля, из равенства нулю следа формы вытекает следуюшее

СлЕДСТВИЕ. Пусть ч есть $\left\{A_{i}\right\}$-мероморфная функиия на кривой $\Lambda$, носитель дивизора которой не пересекается с обгединением множеств $A_{i}$. Пусть $\gamma-$ 1-цепь на кривой $\Lambda$ такая, что дү совпадает с дивизором функиии ч. Тогда для каждой $\left\{A_{i}\right\}$-регулярной формы $\omega$ справедливо соотношение

$$
\int_{\gamma} \omega=0 \bmod (R \omega)
$$

где $R \omega$ - решетка периодов формы $\omega$ на многообразии $\Lambda \backslash\left(\bigcup A_{i}\right)$.

Остановимся на специальном случае. Пусть кривая $\Lambda$ состоит из $n$ экземпляров сферы Римана $\Lambda_{i}$, занумерованных в циклическом порядке. При этом на каждой сфере $\Lambda_{i}$ выделены две различные точки $\left(P_{i}, Q_{i}\right)$. Рассмотрим кривую $\Lambda$ с набором множеств точек $A_{1}, \ldots, A_{n}$, где множество $A_{i}$ при $1 \leqslant i<n$ содержит пару точек $\left(Q_{i}, P_{i+1}\right)$ и множество $A_{n}$ - пару точек $\left(Q_{n}, P_{1}\right)$. Кривую $\Lambda$ с набором $\left\{A_{i}\right\}$ можно себе представить как "алгебраический многоугольник" со "сторонами" $\Lambda_{i}$ и “вершинами” $A_{i}$, в которых склеены точки $Q_{i}$ и $P_{i+1}$.

Примером такого алгебраического многоугольника является нормализация множества одномерных орбит на торической поверхности. Прообраз каждой нульмерной орбиты возникает на нормализации двух одномерных орбит. Эти нормализации надо склеить по прообразам обших нульмерных орбит. 
На алгебраическом многоугольнике $\Lambda$ с точностью до коэффициента существует лишь одна $\left\{A_{i}\right\}$-регулярная форма $\omega$, именно, такая форма $\omega$, что ее ограничение на $\Lambda_{i}$ имеет полюса первого порядка лишш в точках $Q_{i}$ и $P_{i}$, причем ее вычет в точке $Q_{i}$ равен +1 , а вычет в точке $P_{i}$ равен -1 .

Существование одной $\left\{A_{i}\right\}$-мероморфной формы влечет за собой существование одного ограничения на дивизоры $\left\{A_{i}\right\}$-мероморфных функций. Приведем это соотношение. Пусть $t_{i}$ - параметр на сфере $\Lambda_{i}$, имеюший однократньй нуль в точке $Q_{i}$ и однократньй полюс в точке $P_{i}$. Такой параметр единственен с точностью до умножения на ненулевую константу.

Пусть $\varphi-\left\{A_{i}\right\}$-мероморфная функция, $N_{i}, L_{i}-$ множество нулей и полюсов ее ограничения на компоненту $\Lambda_{i}, \mu$ - кратность нуля или полюса, и $\gamma_{i}-1$-цеп, граница $\partial \gamma_{i}$ которой совпадает с дивизором ограничения функции $\varphi$ на компоненту $\Lambda_{i}$.

УТВЕРЖДЕНИЕ 1. ДЛя всякого алгебраического многоугольника $\Lambda$ и всякой $\left\{A_{i}\right\}$-мероморфной функиии на нем справедливо равенство

$$
\prod_{1 \leqslant i \leqslant m} \prod_{a_{j} \in N_{i}}\left(t_{i}\left(a_{j}\right)\right)^{\mu\left(a_{j}\right)}=\prod_{1 \leqslant i \leqslant m} \prod_{b_{j} \in L_{i}}\left(t_{i}\left(b_{j}\right)\right)^{\mu\left(b_{j}\right)} .
$$

ДокАЗАтЕльство. Действительно, по теореме Абеля имеем

$$
\sum_{1 \leqslant i \leqslant m} \int_{\gamma_{i}} \frac{d t_{i}}{t_{i}}=2 k \pi \sqrt{-1}
$$

Интегрируя и потенцируя, получим нужное соотношение.

Утверждение 1 обратимо (чего и следовало ожидать, ведь утверждение 1 - специальньй случай варианта теоремы Абеля).

УТВЕРЖДЕНИЕ 2. Всякий дивизор на алгебраическом многоугольнике $\Lambda$, для которого выполнено соотношение Абеля (1), является дивизором $\left\{A_{i}\right\}$-мероморфной функции.

ДокАЗАТЕЛЬСтво. Ограничение функции $\varphi$ на сферу $\Lambda_{i}$ восстанавливается однозначно с точностью до множителя по ее нулям и полюсам. Вопрос в том, можно ли подобрать эти множители согласованно на всех сферах $\Lambda_{i}$. Восстановим отношение $\varphi\left(P_{i}\right) / \varphi\left(Q_{i}\right)$. Явно вьписьвая рациональную функцию $\varphi$ на сфере $\Lambda_{i}$, получим

$$
\varphi\left(P_{i}\right) \prod_{a_{j} \in N_{i}} t_{i}\left(a_{j}\right)^{k\left(a_{j}\right)}=\varphi\left(Q_{i}\right) \prod_{b_{j} \in L_{i}} t_{i}\left(b_{j}\right)^{k\left(b_{j}\right)} .
$$

Найденные из (2) числа $z_{i}=\varphi\left(P_{i}\right) / \varphi\left(Q_{i}\right)$ связаны соотношением Абеля $\prod z_{i}=1$. Это соотношение и позволяет согласованно восстановить множители на сферах $\Lambda_{i}$ (ср. с доказательством теоремы из §1). 
ЗАмечАниЕ. Соотношение Абеля (1) для алгебраических многоугольников сводится к формуле Виета для произведения корней полинома. Действительно, соотношение (2) есть не что иное, как формула Виета для рациональной функции $\varphi$ на сфере Римана $\Lambda_{i}$. Перемножая эти соотношения по всем сферам, получим соотношение Абеля (1).

Нам понадобится следующая довольно неожиданная

ТеОрема 2. Пусть Г - кривая на торической поверхности $M$, не проходящая через ее неподвижные точки. Тогда найдется линейно әквивалентная ей кривая $\widetilde{\Gamma}$, не проходящая через неподвижные точки, которая пересекается с одномерными орбитами только по точкам с параметром $\xi$, равным (-1).

ДокАЗАТЕльство. Пусть кривая $Г$ задается в $\mathbb{C}^{* 2}$ уравнением $P=0$, и пусть $\Delta$ - многоугольник Ньютона полинома Лорана $P$. Возьмем описанный в следствии из $\S 1$ полином Лорана $Q$, построенньй по многоугольнику $\Delta$. Для этого полинома Лорана $Q$ все полиномы $Q_{\vec{n}}, \vec{n} \in \mathbb{Z}_{\mathrm{ir}}^{2}$, не имеют корней, отличных от $(-1)$. Замыкание $\widetilde{\Gamma}$ кривой, определенной в $\mathbb{C}^{* 2}$ уравнением $Q=0$, обладает требуемыми свойствами. Действительно, дивизор рациональной функции $\Phi=\frac{P}{Q}$ на поверхности $M$ равен $\Gamma-\widetilde{\Gamma}$. (Дивизор не содержит одномерных орбит, так как многоугольники Ньютона полиномов Лорана $P$ и $Q$ равны.) То есть кривые Г и $\widetilde{\Gamma}$ линейно эквивалентны. Далее, кривая $\widetilde{\Gamma}$ пересекает одномерные орбиты лишш в точках с параметром $(-1)$, так как все полиномы $Q_{\vec{n}}$ не имеют корней, отличных от $(-1)$.

Объясним теперь соотношение Виета (см. $\S 4)$, пользуясь теоремой Абеля и теоремой 2. Для данной кривой $Г$, не проходяшей через неподвижные точки торической поверхности, рассмотрим эквивалентную кривую $\widetilde{\Gamma}$, пересекающую одномерные орбиты лиш в точках с параметрами, равными $(-1)$. Такая кривая существует по теореме 2 .

Пусть $\Phi$ - мероморфная функция на торической поверхности $M$, нули которой совпадают с кривой $\Gamma$, а полюса - с кривой $\widetilde{\Gamma}$. Применяя к ограничению $\varphi$ этой функции на объединение одномерных орбит утверждение 1 , получим

$$
\prod_{1 \leqslant i \leqslant m} \prod_{a_{j} \in N_{i}}\left(\xi_{i}\left(a_{j}\right)\right)^{\mu\left(a_{j}\right)}=\prod_{1 \leqslant i \leqslant m}(-1)^{\sum_{a_{j} \in N_{i}} \mu\left(a_{j}\right)} .
$$

Или

$$
\prod\left(-\xi\left(a_{j}\right)\right)^{\mu\left(a_{j}\right)}=1
$$

Мы снова получили соотношение Виета. Итак, с точки зрения теоремы Абеля знак (-1), фигурируюший в этом соотношении, объясняется сушествованием в каждом классе эквивалентности кривой $\widetilde{\Gamma}$, пересекающейся с одномерньми орбитами лишь по точкам, параметры которых равны $(-1)$.

\section{$\S$ 11. Особенности характеристической кривой и многоугольники Ньютона}

Каждой тройке $\Gamma, f, g$ соответствует многоугольник Ньютона $\Delta$ - это многоугольник, построенньй по функции $\mathrm{Mul}=\operatorname{Mul}_{\Gamma f g}(\mathrm{~cm} . \S 1)$. Для всякого многоугольника 
$\Delta$ сушествуют некратные тройки $\Gamma, f, g$, для которых $\Delta$ является многоугольником Ньютона. Ситуация изменится, если наложить ограничение на кривую Г. Ниже приводится необходимое условие на многоугольник $\Delta$, чтобы он был многоугольником Ньютона некоторой некратной тройки $\Gamma, f, g$, в которой $\Gamma$ - связная кривая рода $g$ (см. следствие 1 в этом параграфе). Ограничение на многоугольник $\Delta$ является следствием приводимого ниже вычисления суммы родов особых точек характеристической кривой некратной тройки $\Gamma, f, g$.

Рассмотрим тройку $\Gamma, f, g$, в которой $\Gamma$ - компактная, не обязательно связная кривая и $(f, g)$ - мероморфная вектор-функция. Обозначим через $V \subset \mathbb{Z}_{\mathrm{ir}}^{2}$ множество характеристических векторов функции $\operatorname{Mul}_{\Gamma f g}$. Другими словами, вектор $\vec{n} \in \mathbb{Z}_{\mathrm{ir}}^{2}$ содержится в множестве $V$, если и только если он является типом ростка $(f, g)$ в некоторой точке кривой. Скажем, что торическая поверхность $M \supset \mathbb{C}^{* 2}$ достаточно полная для тройки $\Gamma, f, g$, если веер этой поверхности содержит лучи, порожденные всеми векторами $\vec{n}$ из множества $V$. Обозначим через $\pi: \Gamma \rightarrow \mathbb{C}^{* 2}$ характеристическое мероморфное отображение тройки $\Gamma, f, g$ в тор $\mathbb{C}^{* 2}$, переводяшее точку $a$ в точку $\pi(a)=(f(a), g(a))$.

Лемма 1. Пусть $M \supset \mathbb{C}^{* 2}$ - торическая поверхность, достаточно полная для тройки $\Gamma, f, g$. Тогда характеристическое мероморфное отображение $\pi: \Gamma \rightarrow \mathbb{C}^{* 2}$ продолжается до аналитического отображения $\widetilde{\pi}: \Gamma \rightarrow M$. Образ кривой Г при этом голоморфном отображении не проходит через нульмерные орбиты поверхности $M$.

Доказательство вытекает из утверждения § 3.

Нам понадобятся некоторые инварианты изолированных особых точек (см. [1]). Пусть $P$ - росток голоморфной функции двух переменных, имеющей изолированную особенность в точке $a$. Фиксируем малый шар $B$ с центром в точке $a$. При малых $\varepsilon \neq 0$ уравнение $P=\varepsilon$ определяет в шаре $B$ неособую связную кривую, являюшуюся сферой с $q$ ручками и $k$ дырками. Число дырок $k$ равно числу локально неприводимых ветвей аналитической кривой $P=0$, проходяших через точку $a$. Родом особой точки $a$ кривой $P=0$ называется число $(k-1)+q$.

УТВеРЖДЕНИЕ 1. Род ростка кривой $P=0$ равен $\frac{1}{2}\left(\chi_{0}-\chi_{\varepsilon}\right)$, где $\chi_{0}$ и $\chi_{\varepsilon}-$ әйлеровы характеристики нормализаций кривых, определенных в шаре $B$ уравнениями $P=0$ и $P=\varepsilon$, где $\varepsilon \neq 0$ - достаточно малое комплексное число.

ДокАЗАТЕЛЬСТво. Число $\chi_{0}$ равно числу $k$ локально неприводимых веток кривой $P=0$, проходяших через точку $a$. Число $\chi_{\varepsilon}$ равно $2-2 q-k$. Откуда и вытекает утверждение.

Tеорема 1. Если тройка $\Gamma, f, g$ некратная и соответствующий ей многоугольник Ньютона $\Delta$ двумерен (т.е. не вырождается в отрезок), то сумма родов особых точек замыкания ее характеристической кривой $\pi(\Gamma) \subset \mathbb{C}^{* 2}$ в достаточно полной торической поверхности $M \supset \mathbb{C}^{* 2}$ равна $(K-1)+(B(\Delta)-g)$, әде $K$ - число компонент связности кривой $\Gamma, g$ - ее род и $B(\Delta)$ - число внутренних челых точек в многоугольнике $\Delta$. 
ДокАЗАТЕльство. Рассмотрим уравнение $P=0$ характеристической кривой $\pi(\Gamma) \subset \mathbb{C}^{* 2}$. Многоугольник Ньютона полинома Лорана $P$ совпадает с многоугольником Ньютона тройки $\Gamma, f, g$. Чуть изменим коэффициенты полинома Лорана $P$ так, чтобы полученный при этом полином Лорана $\widetilde{P}$ был $\Delta$-невырожденным для многоугольника $\Delta$ (см. [4]). Согласно теории многогранников Ньютона (см. [5]), если многоугольник Ньютона $\Delta$ двумерен, то замыкание кривой $\widetilde{P}=0$ в достаточно полной торической поверхности будет сферой с числом ручек, равным $B(\Delta)$.

Рассмотрим замыкание в той же торической поверхности исходной характеристической кривой $P=0$. Окружим особые точки $a_{i}$ замькания этой кривой малыми шарами $B_{i}$. Вне объединения шаров $B_{i}$ замыкания кривых $\widetilde{P}=0$ и $P=0$ топологически эквивалентны и имеют одинаковые эйлеровы характеристики. Кривая Г является нормализацией своей характеристической кривой. Согласно утверждению 1 , для шара $B_{i}$ имеем соотношение

$$
\chi\left(\pi^{-1}\left(B_{i}\right)\right)-\chi\left(\widetilde{\Gamma} \cap B_{i}\right)=2 g\left(a_{i}\right),
$$

где $\chi$ - эйлерова характеристика и $g\left(a_{i}\right)$ - род особой точки $a_{i}$ характеристической кривой. Эйлерова характеристика аддитивна. Поэтому сумма разностей $(*)$ эйлеровых характеристик по всем шарам $B_{i}$ равна разности эйлеровых характеристик кривой Г и замыкания кривой $\widetilde{P}=0$, т.е. равна $(2 K-2 g)-(2-2 B(\Delta))$. Теорема 1 доказана.

СЛЕДСТВИЕ 1. На связной кривой Г рода g не существует вектор-функций $(f, g)$, для которых тройка $\Gamma, f, g$ некратна, а многоугольник Ньютона этой тройки двумерен и содержит меньше чем $g$ внутренних чельх точек.

Кривая $\Gamma$, определенная в торе $\mathbb{C}^{* 2}$ общим уравнением $P=0$, с многоугольником Ньютона $\Delta$, вместе с вектор-функцией $(x, y)$ доставляет пример некратной тройки, для которой кривая Г связна и ее род равен числу целых точек внутри многоугольника Ньютона этой тройки.

СлЕДСТВИЕ 2. С точностью до изоморфизма не существует никаких других троек $\Gamma, f, g$, обладающих этим свойством.

СлЕДСТВИЕ 3. Если род связной кривой Г меньше, чем число чельх точек в многоугольнике Ньютона некратной тройки $\Gamma, f, g$, то замыкание характеристической кривой этой тройки в достаточно полной торической поверхности будет иметь особъе точки.

Для достаточно обших троек $\Gamma, f, g$ все особые точки замыкания характеристической кривой лежат в торе $\mathbb{C}^{* 2}$ и не попадают на одномерные орбиты достаточно полной торической поверхности. Опишем явно соответствующие условия обшего положения. Для этого недостаточно знать лишш первые члены разложений функций $f, g$, нужна информация о вторых членах. Рассмотрим росток аналитической кривой $Г$ в точке $a$ с локальным параметром $u, u(a)=0$, и росток вектор-функции $(f, g)$

$$
f=c_{1} u^{b_{1}}\left(1+\rho_{1} u+\cdots\right), \quad g=c_{2} u^{b_{2}}\left(1+\rho_{2} u+\cdots\right),
$$

причем $c_{1} \neq 0, c_{2} \neq 0$ и вектор $\vec{b}=\left(b_{1}, b_{2}\right)$ отличен от нуля. Пусть $\vec{n}-$ тип этого ростка, а $k$-его кратность, т.е. $\vec{n} \in \mathbb{Z}_{\mathrm{ir}}^{2}, \vec{b}=k \vec{n}, k$ - натуральное число. Обозначим 
через $\pi: \Gamma \rightarrow \mathbb{C}^{* 2}$ росток характеристического отображения, через $\tilde{\pi}: \Gamma \rightarrow M-$ его аналитическое продолжение на торическую поверхность, содержащую одномерную орбиту $M_{\infty}^{\vec{n}}$, соответствующую вектору $\vec{n}$.

УТВЕРЖДЕНИЕ 2. Образ ростка $\widetilde{\pi}(\Gamma)$ в поверхности М будет ростком гладкой кривой, трансверсально пересекающей орбиту $M_{\infty}^{\vec{n}}$, если и только если вектор степени $\vec{b}$ неприводим (т.е. кратность $k$ равна 1$)$. Образ ростка $\widetilde{\pi}(\Gamma)$ в поверхности $M$ будет ростком гладкой кривой, касающейся орбиты $M_{\infty}^{\vec{n}}$, если и только если вектор степени $\vec{b}$ приводим (т.е. кратность $k$ больше 1) $u$ коэффициенты $\rho_{1}, \rho_{2}$ не связаны соотношением $b_{1} \rho_{2}=b_{2} \rho_{1}$.

Утверждение проверяется прямым вычислением.

ЗАмЕчАнИЕ. Коэффищиенты $c_{1}, \rho_{1}, c_{2}, \rho_{2}$ зависят от выбора локального параметра $u$. Однако соотношения $c_{1} \neq 0, c_{2} \neq 0, b_{1} \rho_{2} \neq b_{2} \rho_{1}$ инвариантны относительно замены локального параметра.

Будем говорить, что росток $(f, g)$ гладкий на бесконечности, если для него выполнены условия утверждения.

ТЕОРема 2. Если тройка $\Gamma, f, g$ некратная и соответствующий ей многоугольник Ньютона двумерен, то сумма родов особых точек ее характеристической кривой $\pi(\Gamma) \subset \mathbb{C}^{* 2}$ не превосходит числа $(K-1)+(B(\Delta)-g)$, где $K$, $B(\Delta)$ и $g$ такие же, как в теореме 1. Равенство достигается, если и только если все ростки вектор-функиии $(f, g)$ ненулевого типа $\vec{n}$ являются гладкими на бесконечности, и не существует двух различных точек, в которых ростки вектор-функции имеют ненулевой одинаковый тип и одинаковые приведенные числа Вейля.

ДоКАЗАТЕЛЬСТВо.Действительно, род особой точки неотрицателен, и равен нулю лишш для неособой точки. Поэтому неравенство в теореме 2 вытекает из теоремы 1. Неравенство будет равенством, если и только если замькание характеристической кривой неособо в точках его пересечения с одномерными орбитами на поверхности $M$. Для этого нужно, чтобы две гладких ветки не пересекались в одной точке. Для окончания доказательства достаточно воспользоваться утверждением 2 из настоящего параграфа и утверждением 2 из $\S 3$.

Формулируемая ниже теорема 3 (так же как теоремы 1-2) демонстрирует, что многоугольники Ньютона троек $\Gamma, f, g$ играют ту же роль, что и обычные многоугольники Ньютона.

Скажем, что для двух троек $\Gamma_{1}, f_{1}, g_{1}$ и $\Gamma_{2}, f_{2}, g_{2}$ вьполнено условие невырожденности, если не сушествует пары точек $a \in \Gamma_{1}$ и $b \in \Gamma_{2}$, в которых ростки вектор-функции $\left(f_{1}, g_{1}\right)$ и $\left(f_{2}, g_{2}\right)$ имеют одинаковый тип и одинаковые приведенные числа Вейля.

ТеОрема 3. Для любых двух троек $\Gamma_{1}, f_{1}, g_{1} u \Gamma_{2}, f_{2}, g_{2}$ число изолированных точек пересечения их образов при характеристических отображсения, посчитанных с учетом кратностей, не превосходит удвоенного смешанного обгема их многоугольников Ньютона. Равенство достигается, если и только если тройки обладают свойством невьрожденности. 
ДокАЗАТЕЛЬство вытекает из теоремы Бернштейна [2], примененной к характеристическим кривым троек $\Gamma_{1}, f_{1}, g_{1}$ и $\Gamma_{2}, f_{2}, g_{2}$.

\section{СПИСОК ЛИТЕРАТУРЫ}

[1] Арнольд В.И., Варченко А.Н., Гусейн-заде С. М. Особенности дифференцируемых отображений. 1. М.: Наука, 1982.

[2] Бернштейн Д. Н. Число корней системы уравнений // Функцион. анализ и его прилож. 1975. Т. 9. № 3. С. $1-4$.

[3] Данилов В.И. Геометрия торических многообразий // УМН. 1978. $\quad$ Т. 33. № 2. C. $85-135$.

[4] Хованский А.Г. Многогранники Ньютона и торические многообразия // Функцион. анализ и его прилож. 1977. Т. 11. № 4. С. 56-64.

[5] Хованский А. Г. Многогранники Ньютона и родполных пересечений // Функцион. анализ и его прилож. 1978. Т. 12. №1. С. 51-61.

[6] Griffiths Ph. Variations on a theorem of Abel // Invent. Math. 1976. V. 35. №3. P. 321-390.

[7] Kempf G., Knudsen F., Mumford D., Saint-Donat B. Toroidal embeddings // Lecture Notes in Math. V. 339, 1973.

Институт системного анализа РАН;

Поступила в редакцию 